\title{
Simulating the behaviour of Glioblastoma Multiforme based on patient MRI during treatments
}

Flavien Alonzo ( $\sim$ Flavien.Alonzo@ec-nantes.fr )

Ecole Centrale de Nantes https://orcid.org/0000-0002-7972-3126

Mazen Saad

Ecole Centrale de Nantes

Aurelien Serandour

Ecole Centrale de Nantes

\section{Research Article}

Keywords: Glioblastoma Multiforme, Numerical simulations, Treatments model, MRI, Finite Volume

Posted Date: June 24th, 2021

DOI: https://doi.org/10.21203/rs.3.rs-654407/v1

License: (1) This work is licensed under a Creative Commons Attribution 4.0 International License. Read Full License 


\title{
Simulating the behaviour of Glioblastoma Multiforme based on patient MRI during treatments
}

\author{
Flavien Alonzo * Aurelien A. Serandour . \\ Mazen Saad
}

Received: date / Accepted: date

\begin{abstract}
Glioblastoma Multiforme is a brain cancer that still show poor prognosis for patients despite the active researches for new treatments. In this work the goal is to model and simulate the evolution of tumour associated angiogenesis and the therapeutic response of the Glioblastoma Multiforme. Multiple phenomena are modelled in order to fit different biological pathways, such as, the cellular cycle, apoptosis, hypoxia or angiogenesis. This results in a nonlinear system with 4 equations and 4 unknowns: the density of tumour cells, the $\mathrm{O}_{2}$ concentration, the density of endothelial cells and the vascular endothelial growth factor concentration. This system is solved numerically on a 2D-slice of Magnetic Resonance Imaging, using a nonlinear control volume finite element scheme on a mesh fitting the geometry of the brain and the tumour of a patient. We show that this implicit volume finite element numerical scheme is positive and we give energy estimates on the discrete solution to ensure convergence. The numerical scheme is implicit in time. Numerical simulations of this scheme have been done using the different standard treatments: surgery, chemother-
\end{abstract}

Funding: F.A is a recipient of a PhD fellowship from the Ministère de la recherche, A.A.S is supported by chaire INSERM-Ecole Centrale de Nantes, we are also supported by IEA-CNRS and HIPHOP project. Conflicts of interest: none, Availability of data and material: We use MRIs from the patient C3L_16 in the CPTAC-GBM database (TCIA, 2018), Code availability: custom code

Corresponding author: Flavien Alonzo

Ecole Centrale de Nantes, UMR 6629 CNRS, Laboratoire de Mathématiques Jean Leray, F-44321, Nantes, France

Aurelien Serandour

Ecole Centrale de Nantes, F-44000, Nantes, France

Université de Nantes, INSERM, CRCINA, F-44000, Nantes, France

Mazen Saad

Ecole Centrale de Nantes, UMR 6629 CNRS, Laboratoire de Mathématiques Jean Leray, F-44321, Nantes, France

E-mail: Mazen.Saad@ec-nantes.fr 
apy and radiotherapy, in order to understand the behaviour of tumour in response to treatments.

Keywords Glioblastoma Multiforme · Numerical simulations · Treatments model · MRI · Finite Volume

Mathematics Subject Classification (2010) $92-10 \cdot 65 \mathrm{M} 08 \cdot 35 \mathrm{Q} 92$

\section{Introduction}

Glioblastoma Multiforme (GBM) is the deadliest and most frequent brain tumour. Despite the research of new treatments, patients still show poor prognosis in the long run: only $5 \%$ of patients survive 5 years post-prognosis. Usually, patients undergo emergency surgery (if the surgery is possible), then the treatment consists in radiotherapy plus concomitant and adjuvant Temozolomide (TMZ) therapy (Stupp et al, 2005). More efficient therapies remain a major preoccupation to cure GBM, among them, immunotherapies is more and more a subject of research for gliomas (Lim et al, 2018; Kamran et al, 2018) and could improve the current prognosis of GBM patients.

Mathematics have been used for developing models matching the behaviour of gliomas tumour cells in recent years. Some models use a spherical tumour growth approach using Partial Dif-

\section{Tumor-associated angiogenesis}



Fig. 1: Endothelial cells carry blood vessels providing nutrients and $\mathrm{O}_{2}$ in the brain. Due to tumour growth, hypoxic tumour cells are induced by a lack of $\mathrm{O}_{2}$. Hypoxic cells produce proangiogenic factors, mainly Vascular Endothelial Growth Factors, that enhance the formation of new blood vessels. ferential Equations (PDEs)

(Papadogiorgaki et al,

2013; Stein et al, 2007; Kim et al, 2009), other models approach it using an elasticity (Subramanian et al, 2019) or using evolutionary game theoretical model (Basanta et al, 2011). 
When a patient gets diagnosed with GBM, tumour cells have already achieved enough tumour promotion mechanisms in order to evade the immune system and to proliferate in the brain. In that sense, we chose to model the GBM growth based on the process of tumour associated angiogenesis.

Angiogenesis is the ensemble of phenomenon that allow the formation of new blood vessels from pre-existing blood vessels. Those physiological processes happen not only for cancer patients, but tumours have the ability to use angiogenesis in their favor as a tumour promoter (Kim and Lee, 2009). A simplification of the processes used by tumour cells to induce angiogenesis is proposed in figure 1. Tumour cells rely on nutrients and $\mathrm{O}_{2}$ for their growth, provided by blood vessels. During tumour growth, the tumour core lacks $\mathrm{O}_{2}$ inducing hypoxia in the tumour core. Hypoxia prevents most tumour cellular activities, acting like a tumour suppressor process. To fight hypoxia, hypoxic tumour cells produce proangiogenic factors such as, Vascular Endothelial Growth Factors (VEGF) are the main factors produced in GBM. Proangiogenic factors promote angiogenesis meaning that more blood vessels are produced, and so more nutrients and $\mathrm{O}_{2}$ are provided to the tumour cells. Angiogenesis mathematical models have already been developed: using PDEs (Vilanova et al, 2017; Mantzaris et al, 2004; Schugart et al, 2008), some adding stochastic parts in the modeling (Travasso et al, 2011), or working at a mesoscopic scale (Spill et al, 2015). However in this work, we consider more realistic situation to the tumour associated angiogenesis model by working on Magnetic Resonance Imaging (MRIs) data based on a real patient and by modeling the behaviour of GBM growth through the treatments usually administered to patients. Indeed MRIs are required to certify the diagnosis of GBM (Villanueva-Meyer et al, 2017), and it is easier nowadays to get information from MRI as some deep learning techniques can be used to extract medical data (Lundervold and Lundervold, 2019). With tools like CaPTK (Bakas et al, 2017; Pati et al, 2020), it is possible to perform segmentation of GBM tumours based on MRI. Recent studies show also that information on the tumour cells behaviour can be acquired with immunohistochemistry data, for example by identifying GBM subtypes (Orzan et al, 2020) but we will not consider those different subtypes in this work. Working on MRI is numerically challenging because on real MRI we can not have constrained mesh to solve our equations on. Finite volume scheme based on TPFA (Two Point Flux Approximation) can not ensure the positivity of numerical solutions. It is then needed to use more sophisticated numerical schemes in order to ensure the positivity of the solutions. Our approach is based on a CVFE (Control Volume Finite Element) scheme in which nonlinear numerical Gudonov fluxes are used to ensure the positivity.

Using real patient data, it is interesting in the long run to include the treatments in the model to be able to match data and simulations. Currently patients with GBM are treated using surgery, chemotherapy with TMZ and radiotherapy, we will only consider those treatments in our model. Chemotherapy and surgery were first used in PDEs model around gliomas in (Tracqui et al, 1995; Woodward et al, 1996) but more robust models have been developed: on chemotherapy with hypoxic cells (Hinow et al, 2009), on surgery and radiotherapy with an haptotaxis model (Enderling et al, 2010) and even on immunotherapy in gliomas (Banerjee et al, 2015). Choosing to model those treatments will allow us to compare their impact on the GBM growth 
through the recovery of a patient, and so, analyse their advantages and drawbacks on the tumour cells.

\section{The anisotropic degenerate nonlinear angiogenesis model}

Let $\Omega$ be an open bounded polygonal and connected subset of $\mathbb{R}^{2}$ and $T_{f}>0$ a fixed finite time. We denote $\left.Q_{T_{f}}=\Omega \times\right] 0, T_{f}\left[\right.$ and $\left.\Sigma_{T_{f}}=\partial \Omega \times\right] 0, T_{f}[. \Omega$ represents the area in the brain where the tumour is developing, here it is obtained from a slice of an presurgery axial MRI of a patient. $\partial \Omega$ is then the border of the brain around the skull and the ventricles if they are on the MRI (it depends on the location of the tumour). We propose in this work a new model on angiogenesis inspired by works as in (Enderling et al, 2010) and (Hinow et al, 2009), involving reaction-advection-diffusion equations around tumour cells and nutrients. In order to exhibit angiogenesis, two quantity are added into our model: endothelial cells that release $\mathrm{O}_{2}$ in the brain and VEGF (Vascular Endothelial Growth Factor) that are produced by hypoxic tumour cells as a help message that enhance the formation of new endothelial cells. The behaviour of tumour cells during their spread and treatments is chosen to be described by the set of equations

$$
\begin{aligned}
& \partial_{t} u-\nabla \cdot\left(\Lambda_{1}(x)\left(a(u) \nabla u-\chi_{1}(u) \nabla c\right)\right)=\rho_{1} h(c) f_{u_{T}}(u)-\beta_{1} u-T_{\text {treat }}(t, u), \\
& \partial_{t} c-\nabla \cdot\left(D_{2} \nabla c\right)=\alpha_{2} u_{e}-\beta_{2} c-\gamma_{2} u c \\
& \partial_{t} u_{e}-\nabla \cdot\left(\Lambda_{3}(x)\left(a\left(u_{e}\right) \nabla u_{e}-\chi_{3}\left(u_{e}\right) \nabla v\right)\right)=\rho_{3} f_{u_{T}}\left(u_{e}\right)-\beta_{3} u_{e} \\
& \partial_{t} v-\nabla \cdot\left(D_{4} \nabla v\right)=\alpha_{4} g(c) u-\beta_{4} v-\gamma_{4} u_{e} v .
\end{aligned}
$$

We associated with (1a)-(1d) homogeneous zero-flux boundary conditions

$$
\begin{aligned}
& \left(\Lambda_{1}(x) a(u) \nabla u-\Lambda_{1}(x) \chi_{1}(u) \nabla c\right) \cdot \vec{n}=0, \\
& D_{2} \nabla c \cdot \vec{n}=0, \\
& \left(\Lambda_{3}(x) a\left(u_{e}\right) \nabla u_{e}-\Lambda_{3}(x) \chi_{3}\left(u_{e}\right) \nabla v\right) \cdot \vec{n}=0, \\
& D_{4} \nabla v \cdot \vec{n}=0 .
\end{aligned}
$$

These conditions model the no exchange between the brain and the rest of the body. For each quantity, we associated an initial condition on $\Omega$ given by

$$
w(x, t=0)=w_{0}(x), \forall x \in \Omega, w=u, c, u_{e}, v
$$

In the model (1a)-(1d), $u$ is the ratio between the number of tumour cells per $\mathrm{cm}^{2}$ and the maximum tissue capacity $u_{\max }$ ( $u$ is normalized between 0 and 1), $c$ is the concentration in $O_{2}$ in $\mu \mathrm{mol} \cdot \mathrm{cm}^{-2}, u_{e}$ is the ratio between the number of endothelial cells per $\mathrm{cm}^{2}$ and the maximum tissue capacity $u_{\max }\left(u_{e}\right.$ is normalized between 0 and 1) and $v$ is the concentration in Vascular Endothelial Growth Factor (VEGF) in $\mu \mathrm{mol}$. $\mathrm{cm}^{-2}$. The sum of the two cellular populations is $u_{T}=u+u_{e}$. The functions $a(\cdot), \chi \cdot(\cdot)$ and $f_{u_{T}}(\cdot)$ are the cell-density dependant coefficients for diffusion, chemotaxis and growth rate respectively. $g(\cdot)$ is the $\mathrm{O}_{2}$-dependant VEGF production by tumour cells function and $h(\cdot)$ is a $\mathrm{O}_{2}$-dependant threshold allowing mitosis for tumour cells under 
normoxic conditions. $\Lambda_{1}(x)$ and $\Lambda_{3}(x)$ are the medium-dependant diffusion tensor for tumour cells and endothelial cells respectively. The diffusion of cells depends on the white matter, the grey matter and the post-surgical area. $D_{2}$ and $D_{4}$ are the isotropic diffusion tensors associated with $O_{2}$ and VEGF respectively. $\rho_{1}$ is the growth rate of tumour cells, $\alpha_{2}$ is the production rate of $O_{2}$ by endothelial cells, $\rho_{3}$ is the growth rate of endothelial cells and $\alpha_{4}$ is the production rate of VEGF by tumour cells. $\beta_{1}$ and $\beta_{3}$ are the apoptosis rates of tumour cells and endothelial cells respectively, $\beta_{2}$ and $\beta_{4}$ are the degradation rates of $O_{2}$ and VEGF respectively. $\gamma_{2}$ is the consumption rate of $\mathrm{O}_{2}$ by tumour cells and $\gamma_{4}$ is the consumption rate of VEGF by endothelial cells. The map $T_{\text {treat }}(\cdot, \cdot)$ represents the loss of tumour cells due to treatments.

We give the main assumptions of the model (1a)-(3):

(A1) The cell-density diffusion function $a: \mathbb{R} \rightarrow \mathbb{R}^{+}$is a continuous function such that $a(y)>0, \forall y \in] 0,1[$ and $a(y)=0, \forall y \in \mathbb{R} \backslash] 0,1[$.

(A2) The cell-density chemotaxis function $\chi_{m}: \mathbb{R} \rightarrow \mathbb{R}^{+}, m=1,3$ is a continuous function such that $\left.\chi_{m}(y)>0, \forall y \in\right] 0,1\left[\right.$ and $\left.\chi_{m}(y)=0, \forall y \in \mathbb{R} \backslash\right] 0,1[$. Furthermore, we assume there exists a function $\mu_{m} \in \mathscr{C}\left(\mathbb{R}, \mathbb{R}^{+}\right), m=1,3$, such that $\left.\mu_{m}(y)=\frac{a(y)}{\chi_{m}(y)}, \forall y \in\right] 0,1\left[\right.$ and $\left.\mu_{m}(y)=0, \forall y \in \mathbb{R} \backslash\right] 0,1[$.

(A3) The diffusion tensor $\Lambda_{m}(y), m=1,3$ is a bounded, uniformly positive definite tensor on $\Omega$, that is there exists $\Lambda_{m}^{\text {down }}, \Lambda_{m}^{u p}>0$ with

$$
0<\Lambda_{m}^{\text {down }}|y|^{2} \leq\left(\Lambda_{m}(y) y \mid y\right) \leq \Lambda_{m}^{u p}|y|^{2}, m=1,3, \forall y \in \mathbb{R}^{2} \backslash\{0\} .
$$

(A4) All coefficients from (1a)-(1d) are positive

$$
\rho_{1}, \beta_{1}, \alpha_{2}, \beta_{2}, \gamma_{2}, \rho_{3}, \beta_{3}, \alpha_{4}, \beta_{4}, \gamma_{4} \geq 0 .
$$

(A5) The function $f_{u_{T}}(y) \in \mathscr{C}\left(\mathbb{R}, \mathbb{R}^{+}\right)$is

$$
f_{u_{T}}(y)=y\left(1-u_{T}\right)^{+} \mathbb{1}_{[0,1]}(y),
$$

where $x^{+}=\frac{x+|x|}{2}$.

(A6) The initial functions are in $L^{2}(\Omega)$ and follow these inequalities

$$
u_{0}, c_{0}, u_{e_{0}}, v_{0} \geq 0 \text {, a.e. in } \Omega \text { and } u_{0}, u_{e_{0}} \leq 1 \text {, a.e. in } \Omega \text {. }
$$

(A7) $g(\cdot)$ is a piecewise function that allows the production of VEGF by tumour cells only if the tumour cells are in an hypoxic environment and $h(\cdot)$ is the Heaviside step function around the hypoxia threshold

$$
g(y)=y \mathbb{1}_{\left[c_{\text {necro }}, c_{\text {hypo }}\right]}(y), \quad h(y)=H_{c_{\text {hypo }}}(y)=\mathbb{1}_{\left[c_{\text {hypo }},+\infty[\right.}(y) .
$$

$c_{\text {necro }}$ is the threshold under which cells start to necrose and $c_{\text {hypo }}$ is the threshold under which cells lack of $\mathrm{O}_{2}$ to be able to function normally.

(A8) The treatment map $T_{\text {treat }}: \mathbb{R}^{+} \times \mathbb{R} \rightarrow \mathbb{R}^{+}$is positive, piecewise in time and in space. In this work the available treatments are surgery, chemotherapy and radiotherapy. The map $T_{\text {treat }}(\cdot, \cdot)$ models chemotherapy and radiotherapy and can be reconstructed by $T_{\text {treat }}(t, y)=T_{\text {chemo }}(t, y)+T_{\text {radio }}(t, y)$. Moreover $\forall t \in$ $\mathbb{R}^{+}, \forall y \in \mathbb{R}^{-}: T_{\text {treat }}(t, y)=0$. 
2.1 Weak Solution

In order to ensure positivity of the solution in its discrete form we use the following set of functions defined on $\mathbb{R}$

$$
\begin{gathered}
\eta(v)=\max (0, \min (0, v)), \quad p(v)=\int_{1}^{v} \frac{d s}{\eta(s)}, \\
\eta(v) p(v)=0, \forall v \leq 0, \\
\mathscr{A}(v)=\int_{0}^{v} a(s) d s, \quad \xi(v)=\int_{0}^{v} \sqrt{a(s)} d s,
\end{gathered}
$$

for the same ideas as in (Cancès and Guichard, 2016; Cancès et al, 2017; Foucher et al, 2018).

Definition (Weak Solution): Under assumptions (A1)-(A8), we say that the set of measurable functions $\left(u, c, u_{e}, v\right)$ is a weak solution of (1a)-(3) if

$$
\begin{aligned}
& 0 \leq u(t, x), u_{e}(t, x) \leq 1, c(t, x), v(t, x) \geq 0 \text {, for a.e. in } Q_{T_{f}}, \\
& \xi(u) \in L^{2}\left(\left[0, T_{f}\right] ; H^{1}(\Omega)\right) \text {, and } \forall \varphi_{i} \in \mathscr{D}\left(\bar{\Omega} \times\left[0, T_{f}[), i=1, \ldots, 4\right)\right) \text { one has } \\
& \circ-\iint_{Q_{T_{f}}} u \partial_{t} \varphi_{1} d x d t-\int_{\Omega} u_{0}(x) \varphi_{1}^{0} d x \\
& +\iint_{Q_{T_{f}}}\left(\sqrt{a(u)} \Lambda_{1}(x) \nabla \xi(u)-\Lambda_{1}(x) \chi_{1}(u) \nabla c\right) \cdot \nabla \varphi_{1} d x d t \\
& =\iint_{Q_{T_{f}}}\left(\rho_{1} h(c) f_{u_{T}}(u)-\beta_{1} u-T_{\text {treat }}(t, u)\right) \varphi_{1} d x d t, \\
& \circ-\iint_{Q_{T_{f}}} c \partial_{t} \varphi_{2} d x d t-\int_{\Omega} c_{0}(x) \varphi_{2}^{0} d x+\iint_{Q_{T_{f}}} D_{2} \nabla c \cdot \nabla \varphi_{2} d x d t \\
& =\iint_{Q_{T_{f}}}\left(\alpha_{2} u_{e}-\beta_{2} c-\gamma_{2} u c\right) \varphi_{2} d x d t \\
& \circ-\iint_{Q_{T_{f}}} u_{e} \partial_{t} \varphi_{3} d x d t-\int_{\Omega} u_{e_{0}}(x) \varphi_{3}^{0} d x \\
& +\iint_{Q T_{f}}\left(\sqrt{a\left(u_{e}\right)} \Lambda_{3}(x) \nabla \xi\left(u_{e}\right)-\Lambda_{3}(x) \chi_{3}\left(u_{e}\right) \nabla v\right) \cdot \nabla \varphi_{3} d x d t \\
& =\iint_{Q_{T_{f}}}\left(\rho_{3} f_{u_{T}}\left(u_{e}\right)-\beta_{3} u_{e}\right) \varphi_{3} d x d t \\
& \circ-\iint_{Q_{T_{f}}} v \partial_{t} \varphi_{4} d x d t-\int_{\Omega} v_{0}(x) \varphi_{4}^{0} d x+\iint_{Q_{T_{f}}} D_{4} \nabla v \cdot \nabla \varphi_{4} d x d t \\
& =\iint_{Q T_{f}}\left(\alpha_{4} g(c) u-\beta_{4} v-\gamma_{4} u_{e} v\right) \varphi_{4} d x d t .
\end{aligned}
$$




\section{The nonlinear CVFE scheme for system}

The discretization of (1a)-(1d) is chosen following the work of (Foucher et al, 2018), it uses two types of approximations: a conforming FE approximation for diffusion terms, namely second terms in system (1a)-(1d), and a decentered finite volume for haptotaxis terms, namely the third terms in equation (1a) and (1c). The finite element approximation is done over a primal triangular mesh and the finite volume approximation is done over a dual barycentric mesh.

Let $\mathscr{T}$ be a conforming triangulation of the domain $\Omega$, we denote by $\vartheta$ the set of vertices and $\mathscr{E}$ the set of edges in $\mathscr{T} . h_{\mathscr{T}}=\max _{J \in \mathscr{T}} h_{J}$ is the size of the triangulation $\mathscr{T}$, where $h_{J}$ is the diameter of the triangle $J$ and $\theta_{\mathscr{T}}=\max _{I \in \mathscr{T}} \frac{h_{J}}{\rho_{J}}$ is the regularity of the mesh, where $\rho_{J}$ diameter of the incircle of the triangle $J$. For every vertex $K \in \vartheta$, we denote by $x_{K}$ its coordinates, $\mathscr{E}_{K}$ the set of edges having the vertex $K$ as an extremity and $T_{K}$ the set of triangles that have $\mathrm{K}$ as a vertex. If two vertices $K$ and $L$ are joined by an edge then we denote this edge by $\sigma_{K L}$.

For every vertex $K \in \vartheta$, we associate its dual element $\omega_{K}$ constructed by connecting the barycenters of the triangles in $T_{K}$ with the barycenters of the edges in $\mathscr{E}_{K}$, the 2-dimensional Lebesgue measure of $\omega_{K}$ is $m_{K}$. We denote by $\mathscr{M}$ the dual-mesh and $\mathscr{H}_{\mathscr{T}}$ the $\mathbb{P}_{1}$-finite element space on $\Omega$ defined by

$$
\mathscr{H}_{\mathscr{T}}=\left\{\phi \in \mathscr{C}^{0}(\bar{\Omega}): \phi_{\mid J} \in \mathbb{P}_{1}(\mathbb{R}), \forall J \in \mathscr{T}\right\}
$$

We associate $\mathscr{H}_{\mathscr{T}}$ with its canonical basis $\left(\Phi_{K}\right)_{K \in \vartheta}$. Furthermore, we consider the discrete control volume space $\chi_{\mathscr{M}}$ on $\Omega$ defined by

$$
\chi_{\mathscr{M}}=\left\{\phi: \Omega \rightarrow \overline{\mathbb{R}} \text { measurable, } \phi_{\mid \omega_{K}} \text { is constant }, \forall K \in \vartheta\right\}
$$

In this paper, we choose a uniform time discretization with a time step $\delta t=\frac{T_{f}}{N+1}$, where $N$ is a nonnegative integer and we set $t_{n}=n \delta t$, for all $n \in \llbracket 0, N+1 \rrbracket$.

For a given $\left(w_{K}^{n}\right)_{K \in \vartheta, n \in \llbracket 0, N+1 \rrbracket}$, there exists a unique finite element reconstruction $w_{\mathscr{T}, \delta t}$ and a unique constant piecewise reconstruction $w_{\mathscr{M}, \delta t}$ such that

$$
\begin{aligned}
& w_{\mathscr{T}, \delta t}(t, x)=w_{\mathscr{T}}^{n}:=\sum_{K \in \vartheta} w_{K}^{n} \Phi_{K}(x), \quad \forall x \in \Omega, \forall t \in\left(t_{n-1}, t_{n}\right], \\
& w_{\mathscr{M}, \delta t}(t, x)=w_{K}^{n}, \quad \forall x \in \omega_{K}, \forall t \in\left(t_{n-1}, t_{n}\right], \\
& w=u, c, u_{e}, v .
\end{aligned}
$$

The nonlinear CVFE scheme for the discretization of system (1a)-(3) is given by the following set of equations

$$
w_{K}^{0}=\frac{1}{m_{K}} \int_{\omega_{K}} w_{0}(y) d y, \forall K \in \vartheta, \text { with } w=u, c, u_{e}, v,
$$


and $\forall K \in \vartheta, \forall n \in \llbracket 0, N \rrbracket$

$$
\begin{aligned}
& \circ \frac{m_{K}}{\delta t}\left(u_{K}^{n+1}-u_{K}^{n}\right)+\sum_{\sigma_{K L} \in \mathscr{E}_{K}} \Lambda_{K L}^{(1)} a_{K L}^{n+1}\left(u_{K}^{n+1}-u_{L}^{n+1}\right) \\
& -\sum_{\sigma_{K L} \in \mathscr{E}_{K}} \Lambda_{K L}^{(1)} a_{K L}^{n+1} \mu_{K L}^{n+1}\left(c_{K}^{n+1}-c_{L}^{n+1}\right)=m_{K} \rho_{1} h\left(c_{K}^{n+1}\right) f_{u_{T, K}^{n+1}}\left(u_{K}^{n+1}\right) \\
& -m_{K} \beta_{1} u_{K}^{n+1}-m_{K} T_{\text {treat }}\left(t_{n+1}, u_{K}^{n+1}\right), \\
& \circ \frac{m_{K}}{\delta t}\left(c_{K}^{n+1}-c_{K}^{n}\right)+\sum_{\sigma_{K L} \in \mathscr{E}_{K}} D_{K L}^{(2)} \eta_{K L}^{n+1}\left(p\left(c_{K}^{n+1}\right)-p\left(c_{L}^{n+1}\right)\right) \\
& =m_{K} \alpha_{2} u_{e, K}^{n+1}-m_{K} \beta_{2} c_{K}^{n+1}-\gamma_{2} u_{K}^{n+1} c_{K}^{n+1} \text {, } \\
& \circ \frac{m_{K}}{\delta t}\left(u_{e, K}^{n+1}-u_{e, K}^{n}\right)+\sum_{\sigma_{K L} \in \mathscr{E}_{K}} \Lambda_{K L}^{(3)} \tilde{a}_{K L}^{n+1}\left(u_{e, K}^{n+1}-u_{e, L}^{n+1}\right) \\
& -\sum_{\sigma_{K L} \in \mathscr{E}_{K}} \Lambda_{K L}^{(3)} \tilde{a}_{K L}^{n+1} \tilde{\mu}_{K L}^{n+1}\left(v_{K}^{n+1}-v_{L}^{n+1}\right) \\
& =m_{K} \rho_{3} f_{u_{T, K}^{n+1}}\left(u_{e, K}^{n+1}\right)-m_{K} \beta_{3} u_{e, K}^{n+1}, \\
& \circ \frac{m_{K}}{\delta t}\left(v_{K}^{n+1}-v_{K}^{n}\right)+\sum_{\sigma_{K L} \in \mathscr{E}_{K}} D_{K L}^{(4)} \bar{\eta}_{K L}^{n+1}\left(p\left(v_{K}^{n+1}\right)-p\left(v_{L}^{n+1}\right)\right) \\
& =m_{K} \alpha_{4} g\left(c_{K}^{n+1}\right) u_{K}^{n+1}-m_{K} \beta_{4} v_{K}^{n+1}-\gamma_{4} u_{e, K}^{n+1} v_{K}^{n+1} .
\end{aligned}
$$

In the above system, we have used a Finite Element approximation for the diffusion fluxes where the stiffness coefficients are given by

$$
\begin{cases}\Lambda_{K L}^{(m)}=-\int_{\Omega} \Lambda_{m}(x) \cdot \nabla \Phi_{K}(x) \cdot \nabla \Phi_{L}(x) d x & m=1,3, \\ D_{K L}^{(i)}=-\int_{\Omega} D_{i} \nabla \Phi_{K}(x) \cdot \nabla \Phi_{L}(x) d x, & i=2,4 .\end{cases}
$$

We define the intervals

$$
\begin{gathered}
\mathscr{I}_{K L}^{n+1}=\left[\min \left(u_{K}^{n+1}, u_{L}^{n+1}\right), \max \left(u_{K}^{n+1}, u_{L}^{n+1}\right)\right], \\
\square_{K L}^{n+1}=\left[\min \left(u_{e, K}^{n+1}, u_{e, L}^{n+1}\right), \max \left(u_{e, K}^{n+1}, u_{e, L}^{n+1}\right)\right], \\
\mathscr{J}_{K L}^{n+1}=\left[\min \left(c_{K}^{n+1}, c_{L}^{n+1}\right), \max \left(c_{K}^{n+1}, c_{L}^{n+1}\right)\right], \\
\oslash_{K L}^{n+1}=\left[\min \left(v_{K}^{n+1}, v_{L}^{n+1}\right), \max \left(v_{K}^{n+1}, v_{L}^{n+1}\right)\right],
\end{gathered}
$$

to build a Godunov approximation for $a_{K L}^{n+1}, \tilde{a}_{K L}^{n+1}, \eta_{K L}^{n+1}$ and $\bar{\eta}_{K L}^{n+1}$

$$
\begin{aligned}
& a_{K L}^{n+1}=\left\{\begin{array}{ll}
\max _{s \in \mathscr{I}_{K L}^{n+1}} a(s), & \text { if } \Lambda_{K L}^{(1)} \geq 0, \\
\min _{s \in \mathscr{I}_{K L}^{n+1}} a(s), & \text { if } \Lambda_{K L}^{(1)}<0,
\end{array}, \tilde{a}_{K L}^{n+1}= \begin{cases}\max _{s \in \square_{K L}^{n+1}} a(s), & \text { if } \Lambda_{K L}^{(3)} \geq 0, \\
\min _{s \in \square_{K L}^{n+1}} a(s), & \text { if } \Lambda_{K L}^{(3)}<0,\end{cases} \right. \\
& \eta_{K L}^{n+1}=\left\{\begin{array}{ll}
\max _{s \in \mathscr{J}_{K L}^{n+1}} \eta(s), & \text { if } D_{K L}^{(2)} \geq 0, \\
\min _{s \in \mathscr{J}_{K L}^{n+1}} \eta(s), & \text { if } D_{K L}^{(2)}<0,
\end{array}, \bar{\eta}_{K L}^{n+1}= \begin{cases}\max _{s \in \cup_{K L}^{n+1}} \eta(s), & \text { if } D_{K L}^{(4)} \geq 0, \\
\min _{s \in v_{K L}^{n+1}} \eta(s), & \text { if } D_{K L}^{(4)}<0 .\end{cases} \right.
\end{aligned}
$$


Those terms are useful to ensure the positivity of the quantities. The functions $\mu_{1}$ and $\mu_{3}$ are approximated using an upwind scheme

$$
\begin{gathered}
\mu_{K L}^{n+1}= \begin{cases}\mu_{\downarrow}^{(1)}\left(u_{K}^{n+1}\right)+\mu_{\uparrow}^{(1)}\left(u_{L}^{n+1}\right), & \text { if } \Lambda_{K L}^{(1)}\left(c_{K}^{n+1}-c_{L}^{n+1}\right) \geq 0, \\
\mu_{\uparrow}^{(1)}\left(u_{K}^{n+1}\right)+\mu_{\downarrow}^{(1)}\left(u_{L}^{n+1}\right), & \text { if } \Lambda_{K L}^{(1)}\left(c_{K}^{n+1}-c_{L}^{n+1}\right)<0,\end{cases} \\
\tilde{\mu}_{K L}^{n+1}= \begin{cases}\mu_{\downarrow}^{(3)}\left(u_{e, K}^{n+1}\right)+\mu_{\uparrow}^{(3)}\left(u_{e, L}^{n+1}\right), & \text { if } \Lambda_{K L}^{(3)}\left(v_{K}^{n+1}-v_{L}^{n+1}\right) \geq 0, \\
\mu_{\uparrow}^{(3)}\left(u_{e, K}^{n+1}\right)+\mu_{\downarrow}^{(3)}\left(u_{e, L}^{n+1}\right), & \text { if } \Lambda_{K L}^{(3)}\left(v_{K}^{n+1}-v_{L}^{n+1}\right)<0,\end{cases}
\end{gathered}
$$

where the functions $\mu_{\downarrow}^{(1)}, \mu_{\uparrow}^{(1)}, \mu_{\downarrow}^{(3)}$ and $\mu_{\uparrow}^{(3)}$ are given by

$$
\begin{array}{cc}
\mu_{\uparrow}^{(m)}(z)=\int_{0}^{z}\left(\mu_{m}^{\prime}(y)\right)^{+} d y \quad \mu_{\downarrow}^{(m)}(z)=-\int_{0}^{z}\left(\mu_{m}^{\prime}(y)\right)^{-} d y, \quad m=1,3, \\
\forall x \in \mathbb{R}, x=x^{+}-x^{-}, \quad x^{+}=\max (0, x) \text { and } x^{-}=\max (0,-x) .
\end{array}
$$

The description of all variables, coefficients, functions, spaces and mesh components are sum up in the supplementary tables 5-9.

\section{Discrete properties}

4.1 Positivity and upper-boundedness of quantities

Proposition 1 (Positivity of tumour cells concentration) Let's suppose that $u_{K}^{0} \geq 0$ (resp. $\left.u_{e, K}^{0} \geq 0\right)$ for all $K \in \vartheta$, then for all $n \in \llbracket 1, N+1 \rrbracket$ the solution $\left(u_{K}^{n}\right)_{K \in \vartheta}$ of $(12)$ (resp. $\left(u_{e, K}^{n}\right)_{K \in \vartheta}$ of (14)) is positive.

Proof We will show the result only for $u^{n}$ but the same steps are followed for showing the positivity of $u_{e}^{n}$. This proof works by induction on $n$, let's suppose that for a $n \in \llbracket 0, N \rrbracket$ we have $u_{K}^{n} \geq 0, \forall K \in \vartheta$.

Let's $u_{K_{\star}}=u_{K_{\star}}^{n+1}=\min _{M \in \vartheta} u_{M}^{n+1}$ and $\forall L \in \vartheta, u_{L}=u_{L}^{n+1}$. Then by multiplying the equation of (12) associated to $K_{\star}$ by $-u_{K_{\star}}^{-}$, we get

$$
\begin{array}{r}
\frac{m_{K}}{\delta t} u_{K_{\star}}^{-2}+\frac{m_{K}}{\delta t} u_{K}^{n} u_{K_{\star}}^{-}-\sum_{\sigma_{K L} \in \mathscr{E}_{K}} \Lambda_{K L}^{(1)} a_{K L}^{n+1}\left(u_{K_{\star}}-u_{L}\right) u_{K_{\star}}^{-} \\
+\sum_{\sigma_{K L} \in \mathscr{E}_{K}} \Lambda_{K L}^{(1)} a_{K L}^{n+1} \mu_{K L}^{n+1}\left(c_{K}^{n+1}-c_{L}^{n+1}\right) u_{K_{\star}}^{-}=-m_{K} \rho_{1} h\left(c_{K}^{n+1}\right) f_{u_{T, K}^{n+1}}\left(u_{K_{\star}}\right) u_{K_{\star}}^{-} \\
\quad-m_{K} \beta_{1} u_{K_{\star}}^{-2}+m_{K} T_{\text {treat }}\left(t_{n+1}, u_{K_{\star}}\right) u_{K_{\star}}^{-}
\end{array}
$$

but according to assumptions (A5) and (A8), the functions $f_{u_{T}}(\cdot)$ and $T_{\text {treat }}(t, \cdot)$ are extended by zero outside of $[0,1]$, which implies that

$$
f_{u_{T, K}^{n+1}}\left(u_{K_{\star}}\right) u_{K_{\star}}^{-}=0 \text { and } T_{\text {treat }}\left(t_{n+1}, u_{K_{\star}}\right) u_{K_{\star}}^{-}=0 .
$$


Then, if $\Lambda_{K L}^{(1)}<0$ we have $a_{K L}^{n+1} u_{K_{\star}}^{-}=0$ due to the fact that $a(\cdot)$ is extended by zero outside of $[0,1]$, implying that

$$
-\sum_{\sigma_{K L} \in \mathscr{E}_{K}} \Lambda_{K L}^{(1)} a_{K L}^{n+1}\left(u_{K_{\star}}-u_{L}\right) u_{K_{\star}}^{-}=-\sum_{\sigma_{K L} \in \mathscr{E}_{K}} \Lambda_{K L}^{(1)^{+}} a_{K L}^{n+1}\left(u_{K_{\star}}-u_{L}\right) u_{K_{\star}}^{-} .
$$

$\left(u_{K_{\star}}-u_{L}\right) \geq 0$ due to the definition of $u_{K_{\star}}$ and $\Lambda_{K L}^{(1)^{+}} a_{K L}^{n+1} \geq 0$, so we have the positivity of the third term in (21)

$$
-\sum_{\sigma_{K L} \in \mathscr{E}_{K}} \Lambda_{K L}^{(1)} a_{K L}^{n+1}\left(u_{K_{\star}}-u_{L}\right) u_{K_{\star}}^{-} \geq 0 .
$$

For the fourth term in (21) we have

$$
\begin{aligned}
& \mu_{\downarrow}^{(1)}\left(u_{K_{\star}}\right)+\mu_{\uparrow}^{(1)}\left(u_{L}\right)=\mu_{1}\left(u_{K_{\star}}\right)-\mu_{1}(0)+\int_{u_{K_{\star}}}^{u_{L}}\left(\mu_{1}^{\prime}(s)\right)^{+} d s, \\
& \mu_{\uparrow}^{(1)}\left(u_{K_{\star}}\right)+\mu_{\downarrow}^{(1)}\left(u_{L}\right)=\mu_{1}\left(u_{K_{\star}}\right)-\mu_{1}(0)-\int_{u_{K_{\star}}}^{u_{L}}\left(\mu_{1}^{\prime}(s)\right)^{-} d s,
\end{aligned}
$$

so, since $u_{K_{\star}} \leq u_{L}$, we deduce that

$$
\begin{aligned}
& \left(\mu_{\downarrow}^{(1)}\left(u_{K_{\star}}\right)+\mu_{\uparrow}^{(1)}\left(u_{L}\right)\right) u_{K_{\star}}^{-}=+u_{K_{\star}}^{-} \int_{u_{K_{\star}}}^{u_{L}}\left(\mu_{1}^{\prime}(s)\right)^{+} d s \geq 0, \\
& \left(\mu_{\uparrow}^{(1)}\left(u_{K_{\star}}\right)+\mu_{\downarrow}^{(1)}\left(u_{L}\right)\right) u_{K_{\star}}^{-}=-u_{K_{\star}}^{-} \int_{u_{K_{\star}}}^{u_{L}}\left(\mu_{1}^{\prime}(s)\right)^{-} d s \leq 0 .
\end{aligned}
$$

According to the definitions $a_{K L}^{n+1}$ in (17) and $\mu_{K L}^{n+1}$ in (19), we have $\Lambda_{K L}^{(1)} a_{K L}^{n+1} \mu_{K L}^{n+1}\left(c_{K}^{n+1}-\right.$ $\left.c_{L}^{n+1}\right) u_{K_{\star}}^{-} \geq 0$ whenever the sign of $\Lambda_{K L}^{(1)}\left(c_{K}^{n+1}-c_{L}^{n+1}\right)$, which gives the positivity of the fourth term in (21)

$$
+\sum_{\sigma_{K L} \in \mathscr{E}_{K}} \Lambda_{K L}^{(1)} a_{K L}^{n+1} \mu_{K L}^{n+1}\left(c_{K}^{n+1}-c_{L}^{n+1}\right) u_{K_{\star}}^{-} \geq 0 .
$$

With inequalities (22) and (23), we can then conclude that the left hand side of (21) is positive. However

$$
\begin{aligned}
\frac{m_{K}}{\delta t} u_{K_{\star}}^{-2}+\frac{m_{K}}{\delta t} u_{K}^{n} u_{K_{\star}}^{-} & -\sum_{\sigma_{K L} \in \mathscr{E}_{K}} \Lambda_{K L}^{(1)} a_{K L}^{n+1}\left(u_{K_{\star}}-u_{L}\right) u_{K_{\star}}^{-} \\
& +\sum_{\sigma_{K L} \in \mathscr{E}_{K}} \Lambda_{K L}^{(1)} a_{K L}^{n+1} \mu_{K L}^{n+1}\left(c_{K}^{n+1}-c_{L}^{n+1}\right) u_{K_{\star}}^{-}=-m_{K} \beta_{1} u_{K_{\star}}^{-2} \leq 0,
\end{aligned}
$$

So all the terms on the left-side of the above inequality are null because they were non-negative, implying that $\frac{m_{K}}{\delta t} u_{K_{\star}}^{-2}=0$, giving the result from the proposition.

Proposition 2 (Boundedness of tumour cells concentration) Let's suppose that $0 \leq u_{K}^{0} \leq 1$ (resp. $\left.0 \leq u_{e, K}^{0} \leq 1\right)$ for all $K \in \vartheta$, then for all $n \in \llbracket 1, N+1 \rrbracket$ the solution $\left(u_{K}^{n}\right)_{K \in \vartheta}$ of (12) (resp. $\left(u_{e, K}^{n}\right)_{K \in \vartheta}$ of (14)) is upper-bounded by 1. 
Proof We will show the result only for $u^{n}$ but the same steps are followed for showing that $u_{e}^{n}$ upper-bounded. This proof works by induction on $n$, let's suppose that for a $n \in \llbracket 0, N \rrbracket$ we have $u_{K}^{n} \leq 1, \forall K \in \vartheta$.

Let's $u_{K^{\star}}=u_{K}^{n+1}=\max _{H \in \vartheta} u_{H}^{n+1}$ and $\forall L \in \vartheta, u_{L}=u_{L}^{n+1}$. Then by multiplying the equation of (12) associated with $K^{\star}$ by $\left(1-u_{K^{\star}}\right)^{-}$, we get

$$
\begin{aligned}
\frac{m_{K}}{\delta t}\left|\left(1-u_{K^{\star}}\right)^{-}\right|^{2}+ & \frac{m_{K}}{\delta t}\left(1-u_{K}^{n}\right)\left(1-u_{K^{\star}}\right)^{-} \\
& +\sum_{\sigma_{K L} \in \mathscr{E}_{K}} \Lambda_{K L}^{(1)} a_{K L}^{n+1}\left(u_{K^{\star}}-u_{L}\right)\left(1-u_{K^{\star}}\right)^{-} \\
- & \sum_{\sigma_{K L} \in \mathscr{E}_{K}} \Lambda_{K L}^{(1)} a_{K L}^{n+1} \mu_{K L}^{n+1}\left(c_{K}^{n+1}-c_{L}^{n+1}\right)\left(1-u_{K^{\star}}\right)^{-} \\
& =m_{K} \rho_{1} h\left(c_{K}^{n+1}\right) f_{u_{T K}^{n+1}}\left(u_{K^{\star}}\right)\left(1-u_{K^{\star}}\right)^{-} \\
& -m_{K} \beta_{1} u_{K^{\star}}\left(1-u_{K^{\star}}\right)^{-}-m_{K} T_{\text {treat }}\left(t_{n+1}, u_{K^{\star}}\right)\left(1-u_{K^{\star}}\right)^{-} .
\end{aligned}
$$

Using proposition 1 , we know that $u_{K^{\star}} \geq u_{L} \geq 0, \forall L \in \vartheta$. If $\Lambda_{K L}^{(1)}<0$, we have $a_{K L}^{n+1}\left(1-u_{K^{\star}}\right)^{-}=0$ due to the fact that $a(\cdot)$ is set to zero outside of $[0,1]$. So knowing that $a_{K L}^{n+1} \geq 0$, we have

$$
\Lambda_{K L}^{(1)} a_{K L}^{n+1}\left(1-u_{K^{\star}}\right)^{-} \geq 0,
$$

then observing that $\left(u_{K^{\star}}-u_{L}\right) \geq 0$, we conclude that the third term in (24) is positive

$$
+\sum_{\sigma_{K L} \in \mathscr{E}_{K}} \Lambda_{K L}^{(1)} a_{K L}^{n+1}\left(u_{K^{\star}}-u_{L}\right)\left(1-u_{K^{\star}}\right)^{-} \geq 0
$$

The function $f_{u_{T}}(\cdot)$ is extended by zero outside of $[0,1]$, implying that $f_{u_{T, K}^{n+1}}\left(u_{K^{\star}}\right)(1-$ $\left.u_{K^{\star}}\right)^{-}=0$. Since $\left(u_{L} \leq u_{K^{\star}}\right)$ and

$$
\begin{aligned}
& \mu_{\downarrow}^{(1)}\left(u_{K^{\star}}\right)+\mu_{\uparrow}^{(1)}\left(u_{L}\right)=\mu_{1}\left(u_{K^{\star}}\right)-\mu_{1}(0)-\int_{u_{L}}^{u_{K^{\star}}}\left(\mu_{1}^{\prime}(s)\right)^{+} d s, \\
& \mu_{\uparrow}^{(1)}\left(u_{K^{\star}}\right)+\mu_{\downarrow}^{(1)}\left(u_{L}\right)=\mu_{1}\left(u_{K^{\star}}\right)-\mu_{1}(0)+\int_{u_{L}}^{u_{K^{\star}}}\left(\mu_{1}^{\prime}(s)\right)^{-} d s,
\end{aligned}
$$

we have

$$
\begin{aligned}
& \left(\mu_{\downarrow}^{(1)}\left(u_{K^{\star}}\right)+\mu_{\uparrow}^{(1)}\left(u_{L}\right)\right)\left(1-u_{K^{\star}}\right)^{-}=-\left(1-u_{K^{\star}}\right)^{-} \int_{u_{L}}^{u_{K^{\star}}}\left(\mu_{1}^{\prime}(s)\right)^{+} d s \leq 0, \\
& \left(\mu_{\uparrow}^{(1)}\left(u_{K^{\star}}\right)+\mu_{\downarrow}^{(1)}\left(u_{L}\right)\right)\left(1-u_{K^{\star}}\right)^{-}=+\left(1-u_{K^{\star}}\right)^{-} \int_{u_{L}}^{u_{K^{\star}}}\left(\mu_{1}^{\prime}(s)\right)^{-} d s \geq 0 .
\end{aligned}
$$

So whenever the sign of $\Lambda_{K L}^{(1)}\left(c_{K}^{n+1}-c_{L}^{n+1}\right)$

$$
\Lambda_{K L}^{(1)}\left(c_{K}^{n+1}-c_{L}^{n+1}\right) \mu_{K L}^{n+1}\left(1-u_{K^{\star}}\right)^{-} \leq 0,
$$


and, having $a_{K L}^{n+1} \geq 0$, we conclude that the fourth term in (24) is positive

$$
-\sum_{\sigma_{K L} \in \mathscr{E}_{K}} \Lambda_{K L}^{(1)} a_{K L}^{n+1} \mu_{K L}^{n+1}\left(c_{K}^{n+1}-c_{L}^{n+1}\right)\left(1-u_{K^{\star}}\right)^{-} \geq 0 .
$$

With (25), (26) and the positivity of $T_{\text {treat }}(\cdot, \cdot)$, we conclude also that

$$
\begin{aligned}
\frac{m_{K}}{\delta t}\left|\left(1-u_{K^{\star}}\right)^{-}\right|^{2} & +\frac{m_{K}}{\delta t}\left(1-u_{K}^{n}\right)\left(1-u_{K^{\star}}\right)^{-}+\sum_{\sigma_{K L} \in \mathscr{E}_{K}} \Lambda_{K L}^{(1)} a_{K L}^{n+1}\left(u_{K^{\star}}-u_{L}\right)\left(1-u_{K^{\star}}\right)^{-} \\
& -\sum_{\sigma_{K L} \in \mathscr{E}_{K}} \Lambda_{K L}^{(1)} a_{K L}^{n+1} \mu_{K L}^{n+1}\left(c_{K}^{n+1}-c_{L}^{n+1}\right)\left(1-u_{K^{\star}}\right)^{-} \\
& =-m_{K} \beta_{1} u_{K^{\star}}\left(1-u_{K^{\star}}\right)^{-}-m_{K} T_{\text {treat }}\left(t_{n+1}, u_{K^{\star}}\right)\left(1-u_{K^{\star}}\right)^{-} \leq 0 .
\end{aligned}
$$

So all terms on left side of (24) being non-negative, they are null, in particular: $\frac{m_{K}}{\delta t}\left(1-u_{K^{\star}}\right)^{-2}=0$, then $\left(1-u_{K^{\star}}\right)^{-}=0$, which gives the result of this proposition.

Proposition 3 (Positivity of $O_{2}$ and VEGF) Let's suppose that $c_{K}^{0} \geq 0$ (resp. $\left.v_{K}^{0} \geq 0\right)$ for all $K \in \vartheta$, then for all $n \in \llbracket 0, N+1 \rrbracket$ the solution $\left(c_{K}^{n}\right)_{K \in \vartheta}$ of (13) (resp. $\left(v_{K}^{n}\right)_{K \in \vartheta}$ of (15)) is positive.

Proof We will show the result only for $c^{n}$ but the same steps are followed for showing the positivity of $v^{n}$. This proof works by induction on $n$, let's suppose that for a $n \in \llbracket 0, N \rrbracket$ we have $c_{K}^{n} \geq 0, \forall K \in \vartheta$.

Let's $c_{K_{\star}}=c_{K}^{n+1}=\min _{H \in \vartheta} c_{H}^{n+1}$ and $\forall L \in \vartheta, c_{L}=c_{L}^{n+1}$. Then by multiplying the equation of (13) associated with $K_{\star}$ by $-c_{K_{\star}}^{-}$, we get

$$
\begin{aligned}
\frac{m_{K}}{\delta t} c_{K_{\star}}^{-2}+\frac{m_{K}}{\delta t} c_{K_{\star}}^{-} c_{K}^{n}-\sum_{\sigma_{K L} \in \mathscr{E}_{K}} D_{K L}^{(2)} \eta_{K L}^{n+1}\left(p\left(c_{K_{\star}}\right)-p\left(c_{L}\right)\right) c_{K_{\star}}^{-} \\
=-m_{K} \alpha_{2} u_{e}^{n+1} c_{K_{\star}}^{-}-m_{K} \beta_{2} c_{K_{\star}}^{-2}-\gamma_{2} u_{K}^{n+1} c_{K_{\star}}^{-2} .
\end{aligned}
$$

Using the results of proposition 1 and 2, we have $u_{e}^{n+1}, u_{K}^{n+1} \geq 0$, implying that

$$
-m_{K} \alpha_{2} u_{e}^{n+1} c_{K_{\star}}^{-} \leq 0, \quad-m_{K} \beta_{2} c_{K_{\star}}^{-2} \leq 0 \quad \text { and } \quad-\gamma_{2} u_{K}^{n+1} c_{K_{\star}}^{-2} \leq 0 .
$$

If $D_{K L}^{(2)}<0$ then $\eta_{K L}^{n+1} c_{K_{\star}}^{-}=0$. Observing that $\mu_{K L}^{n+1} \geq 0$ and that the function $p(\cdot)$ is non-decreasing on $\mathbb{R}$, we have $\left(p\left(c_{K_{\star}}\right)-p\left(c_{L}\right)\right) \leq 0$, meaning that

$$
\begin{aligned}
-\sum_{\sigma_{K L} \in \mathscr{E}_{K}} D_{K L}^{(2)} \eta_{K L}^{n+1}\left(p\left(c_{K_{\star}}\right)\right. & \left.-p\left(c_{L}\right)\right) c_{K_{\star}}^{-} \\
& =-\sum_{\sigma_{K L} \in \mathscr{E}_{K}}\left(D_{K L}^{(2)}\right)^{+} \eta_{K L}^{n+1}\left(p\left(c_{K_{\star}}\right)-p\left(c_{L}\right)\right) c_{K_{\star}}^{-} \geq 0 .
\end{aligned}
$$

Then

$$
\begin{aligned}
& \frac{m_{K}}{\delta t} c_{K_{\star}}^{-2}+\frac{m_{K}}{\delta t} c_{K_{\star}}^{-} c_{K}^{n}-\sum_{\sigma_{K L} \in \mathscr{E}_{K}} D_{K L}^{(2)} \eta_{K L}^{n+1}\left(p\left(c_{K_{\star}}\right)-p\left(c_{L}\right)\right) c_{K_{\star}}^{-} \\
& =-m_{K} \alpha_{2} u_{e}^{n+1} c_{K_{\star}}^{-}-m_{K} \beta_{2} c_{K_{\star}}^{-2}-\gamma_{2} u_{K}^{n+1} c_{K_{\star}}^{-2} \leq 0 .
\end{aligned}
$$


The terms on the left-side of (27) being non-negative, we conclude that they are all null. In particular $c_{K_{\star}}^{-2}=0$, which completes the proof of the proposition.

Remark 1 Notice that we could consider a semi-implicit scheme by replacing $c^{n+1}$ by $c^{n}$ in (12), $u^{n+1}$ by $u^{n}$ in (13) and (15), $v^{n+1}$ by $v^{n}$ in (14), $u_{e}^{n+1}$ by $u_{e}^{n}$ in (13) and (15) and the propositions 1,2 and 3 would still hold.

\subsection{Algorithm to get the discrete solution (12)-(15)}

In this work, we propose an iterative algorithm in order to get the discrete solution of the implicit numerical scheme (12)-(15). The main idea of this algorithm is to solve the nonlinear system (12)-(15) with an iterative method that has a simplest numerical complexity and that converges to the solution of the implicit numerical scheme. Let's suppose that $\nabla c_{0}$ and $\nabla v_{0}$ are in $L^{2}(\Omega)$ and consider a given solution $\Pi_{\mathscr{T}}^{n}=\left(u_{K}^{n}, c_{K}^{n}, u_{e, K}^{n}, v_{K}^{n}\right)_{K \in \vartheta}$ for a given $n$. We look for the solution $\Pi_{\mathscr{T}}^{n+1}$ at $t_{n+1}$ as the limit of the following iterative process when $m \rightarrow+\infty$ :

Initialisation: $w_{K}^{(0)}=w_{K}^{n}, \forall K \in \vartheta, w=u, c, u_{e}, v$.

For $m \geq 0$,

$$
\begin{aligned}
& \circ \frac{m_{K}}{\delta t}\left(u_{K}^{m+1}-u_{K}^{(0)}\right)+\sum_{\sigma_{K L} \in \mathscr{E}_{K}} \Lambda_{K L}^{(1)} a_{K L}^{m+1}\left(u_{K}^{m+1}-u_{L}^{m+1}\right) \\
& -\sum_{\sigma_{K L} \in \mathscr{E}_{K}} \Lambda_{K L}^{(1)} a_{K L}^{m+1} \mu_{K L}^{m+1}\left(c_{K}^{m}-c_{L}^{m}\right) \\
& =m_{K} h\left(c_{K}^{m}\right) \rho_{1} f_{u_{T, K}^{m}}\left(u_{K}^{m+1}\right)-m_{K} \beta_{1} u_{K}^{m+1}-m_{K} T_{\text {treat }}\left(t_{m+1}, u_{K}^{m+1}\right), \\
& \circ \frac{m_{K}}{\delta t}\left(c_{K}^{m+1}-c_{K}^{(0)}\right)+\sum_{\sigma_{K L} \in \mathscr{E}_{K}} D_{K L}^{(2)} \eta_{K L}^{m+1}\left(p\left(c_{K}^{m+1}\right)-p\left(c_{L}^{m+1}\right)\right) \\
& =m_{K} \alpha_{2} u_{e, K}^{m}-m_{K} \beta_{2} c_{K}^{m+1}-\gamma_{2} u_{K}^{m} c_{K}^{m+1}, \\
& \circ \frac{m_{K}}{\delta t}\left(u_{e, K}^{m+1}-u_{e, K}^{(0)}\right)+\sum_{\sigma_{K L} \in \mathscr{E}_{K}} \Lambda_{K L}^{(3)} \tilde{a}_{K L}^{m+1}\left(u_{e, K}^{m+1}-u_{e, L}^{m+1}\right) \\
& -\sum_{\sigma_{K L} \in \mathscr{E}_{K}} \Lambda_{K L}^{(3)} \tilde{a}_{K L}^{m+1} \tilde{\mu}_{K L}^{m+1}\left(v_{K}^{m}-v_{L}^{m}\right) \\
& =m_{K} \rho_{3} f_{u_{T, K}^{m}}\left(u_{e, K}^{m+1}\right)-m_{K} \beta_{3} u_{e, K}^{m+1}, \\
& \circ \frac{m_{K}}{\delta t}\left(v_{K}^{m+1}-v_{K}^{(0)}\right)+\sum_{\sigma_{K L} \in \mathscr{E}_{K}} D_{K L}^{(4)} \bar{\eta}_{K L}^{m+1}\left(p\left(v_{K}^{m+1}\right)-p\left(v_{L}^{m+1}\right)\right) \\
& =m_{K} \alpha_{4} g\left(c_{K}^{m}\right) u_{K}^{m}-m_{K} \beta_{4} v_{K}^{m+1}-\gamma_{4} u_{e, K}^{m} v_{K}^{m+1} .
\end{aligned}
$$

Observe that at each iteration $m$, the system (29)-(32) is non-coupled, its resolution consists of four independent equations which is easier to solve than (12)-(15).

Let $\tau^{(n)}: \tilde{X}=\left(\tilde{u}, \tilde{c}, \tilde{u}_{e}, \tilde{v}\right) \mapsto X=\left(u, c, u_{e}, v\right)$ be the application that for the vector $\tilde{X}=\left(\tilde{u}, \tilde{c}, \tilde{u}_{e}, \tilde{v}\right) \in\left(\mathbb{R}^{\# \vartheta}\right)^{4}$ associates the solution $X=\left(u, c, u_{e}, v\right)$ of (29)-(32). Replacing $\left(u^{m}, c^{m}, u_{e}^{m}, v^{m}\right)$ by $\tilde{X}$ and $\left(u^{m+1}, c^{m+1}, u_{e}^{m+1}, v^{m+1}\right)$ by $X$, the iterative method is 
equivalent to

$$
\begin{cases}X_{m+1} & =\tau^{(n)}\left(X_{m}\right), \\ X_{0} & \in E=\left\{(w, x, y, z) \in\left(\mathbb{R}^{\# \vartheta}\right)^{4}: 0 \leq w, y \leq 1 \text { and } 0 \leq x, z\right\} .\end{cases}
$$

Note that if the sequence $\left(X_{m}\right)_{m}$ converges, then $X_{m} \underset{m \rightarrow \infty}{\rightarrow} \Pi_{\mathscr{T}}^{n+1}$.

Let's remember that $0 \leq u_{K}^{n}, u_{e, K}^{n} \leq 1$ and $c_{K}^{n}, v_{K}^{n} \geq 0$ for all $K \in \vartheta$ and $n \geq 0$, so the solutions of the iterative method follow $0 \leq u^{m}, u_{e}^{m} \leq 1$ and $c^{m}, v^{m} \geq 0$ for all $m \geq 0$ due to Proposition 1-3 and Remark 1.

To prove the existence of the solution of (29)-(32) and that the application $\tau^{(n)}$ is well defined, we will use the following energy estimates on the iterative method.

Lemma 1 There exists $C_{1}\left(\Lambda_{1}, \theta_{\mathscr{T}}\right), C_{2}\left(D_{2}, \theta_{\mathscr{T}}\right)>0$ such that:

$$
\begin{gathered}
\sum_{\sigma_{K L} \in \mathscr{E}}\left|\Lambda_{K L}^{(1)}\right| a_{K L}^{m+1}\left(u_{K}^{m+1}-u_{L}^{m+1}\right)^{2} \leq C_{1} \sum_{\sigma_{K L} \in \mathscr{E}} \Lambda_{K L}^{(1)} a_{K L}^{m+1}\left(u_{K}^{m+1}-u_{L}^{m+1}\right)^{2}, \\
\sum_{\sigma_{K L} \in \mathscr{E}}\left|D_{K L}^{(2)}\right| \eta_{K L}^{m+1}\left(p\left(c_{K}^{m+1}\right)-p\left(c_{L}^{m+1}\right)\right)^{2} \\
\leq C_{2} \sum_{\sigma_{K L} \in \mathscr{E}} D_{K L}^{(2)} \eta_{K L}^{m+1}\left(p\left(c_{K}^{m+1}\right)-p\left(c_{L}^{m+1}\right)\right)^{2} .
\end{gathered}
$$

Proof This comes from Lemma 3.1-3.3 in (Cancès and Guichard, 2016). $\left(u_{e_{K}}^{m+1}\right)_{K}$ (resp. $\left.\left(v_{K}^{m+1}\right)_{K}\right)$ follows the same inequality as $\left(u_{K}^{m+1}\right)_{K}$ (resp. $\left.\left(c_{K}^{m+1}\right)_{K}\right)$ with a constant $C_{3}\left(\Lambda_{3}, \theta_{\mathscr{T}}\right)\left(\right.$ resp. $\left.C_{4}\left(D_{4}, \theta_{\mathscr{T}}\right)\right)$.

Lemma 2 There exists $C_{5}\left(D_{2}, \theta_{\mathscr{T}}\right), C_{6}\left(D_{4}, \theta_{\mathscr{T}}\right)$ such that

$$
\begin{aligned}
& \sum_{\sigma_{K L} \in \mathscr{E}}\left|D_{K L}^{(2)}\right| \eta_{K L}^{m+1}(\left.p\left(c_{K}^{m+1}\right)-p\left(c_{L}^{m+1}\right)\right)\left(c_{K}^{m+1}-c_{L}^{m+1}\right) \\
& \leq C_{5} \sum_{\sigma_{K L} \in \mathscr{E}} D_{K L}^{(2)} \eta_{K L}^{m+1}\left(p\left(c_{K}^{m+1}\right)-p\left(c_{L}^{m+1}\right)\right)\left(c_{K}^{m+1}-c_{L}^{m+1}\right), \\
& \sum_{\sigma_{K L} \in \mathscr{E}}\left|D_{K L}^{(4)}\right| \bar{\eta}_{K L}^{m+1}\left(p\left(v_{K}^{m+1}\right)-p\left(v_{L}^{m+1}\right)\right)\left(v_{K}^{m+1}-v_{L}^{m+1}\right) \\
& \leq C_{6} \sum_{\sigma_{K L} \in \mathscr{E}} D_{K L}^{(4)} \bar{\eta}_{K L}^{m+1}\left(p\left(v_{K}^{m+1}\right)-p\left(v_{L}^{m+1}\right)\right)\left(v_{K}^{m+1}-v_{L}^{m+1}\right) .
\end{aligned}
$$

Proof The proof relies on the fact that

$$
\begin{aligned}
\sum_{\sigma_{K L} \in \mathscr{E}}\left|D_{K L}^{(2)}\right| \eta_{K L}^{m+1}\left(p\left(c_{K}^{m+1}\right)-p\left(c_{L}^{m+1}\right)\right)\left(c_{K}^{m+1}-c_{L}^{m+1}\right) \\
=\sum_{\substack{\sigma_{K L} \in \mathscr{E} \\
p\left(c_{K}^{m+1}\right) \neq p\left(c_{L}^{m+1}\right)}}\left|D_{K L}^{(2)}\right| \eta_{K L}^{m+1}\left(p\left(c_{K}^{m+1}\right)-p\left(c_{L}^{m+1}\right)\right)^{2} \frac{\left(c_{K}^{m+1}-c_{L}^{m+1}\right)}{\left(p\left(c_{K}^{m+1}\right)-p\left(c_{L}^{m+1}\right)\right)},
\end{aligned}
$$


and that the quantity $\frac{\left(c_{K}^{m+1}-c_{L}^{m+1}\right)}{\left(p\left(c_{K}^{m+1}\right)-p\left(c_{L}^{m+1}\right)\right)}$ is nonnegative because $p(\cdot)$ is non decreasing, we can then use the same arguments than Lemma 3.1-3.3 in (Cancès and Guichard, 2016) to show that

$$
\begin{gathered}
\sum_{\substack{\sigma_{K L} \in \mathscr{E} \\
p\left(c_{K}^{m+1}\right) \neq p\left(c_{L}^{m+1}\right)}}\left|D_{K L}^{(2)}\right| \eta_{K L}^{m+1}\left(p\left(c_{K}^{m+1}\right)-p\left(c_{L}^{m+1}\right)\right)^{2} \frac{\left(c_{K}^{m+1}-c_{L}^{m+1}\right)}{\left(p\left(c_{K}^{m+1}\right)-p\left(c_{L}^{m+1}\right)\right)} \\
\leq C_{5} \sum_{\substack{\sigma_{K L} \in \mathscr{E} \\
p\left(c_{K}^{m+1}\right) \neq p\left(c_{L}^{m+1}\right)}} D_{K L}^{(2)} \eta_{K L}^{m+1}\left(p\left(c_{K}^{m+1}\right)-p\left(c_{L}^{m+1}\right)\right)^{2} \frac{\left(c_{K}^{m+1}-c_{L}^{m+1}\right)}{\left(p\left(c_{K}^{m+1}\right)-p\left(c_{L}^{m+1}\right)\right)} .
\end{gathered}
$$

The same arguments are used for the inequality in $v$.

Proposition 4 (Existence of a discrete solution) For $n \in \llbracket 0, N \rrbracket$ and $\forall m \geq 0$ there exists a solution $\left(u^{m+1}, c^{m+1}, u_{e}^{m+1}, v^{m+1}\right)$ from equations (29)-(32) with $0 \leq u^{m+1}, u_{e}^{m+1} \leq$ 1 and $c^{m+1}, v^{m+1} \geq 0$

Proof This works by induction on $m$.

Let's consider the application: $\mathscr{W}=\left(\mathscr{W}_{u}, \mathscr{W}_{c}, \mathscr{W}_{u_{e}}, \mathscr{W}_{v}\right):\left(\mathbb{R}^{\# \vartheta}\right)^{4} \rightarrow\left(\mathbb{R}^{\# \vartheta}\right)^{4}$ where $\forall K \in$ $\vartheta$, for $n \in \llbracket 0, N \rrbracket$ and $\forall m \geq 0$

$$
\begin{aligned}
& \circ\left(\mathscr{W}_{u}(y)\right)_{K}=m_{K} \frac{y_{K}-u_{K}^{n}}{\delta t}+\sum_{\sigma_{K L} \in \varepsilon_{K}} \Lambda_{K L}^{(1)} a_{K L}^{m+1}\left(y_{K}-y_{L}\right) \\
& -\sum_{\sigma_{K L} \in \varepsilon_{K}} \Lambda_{K L}^{(1)} a_{K L}^{m+1} \mu_{K L}^{m+1}\left(c_{K}^{m}-c_{L}^{m}\right)-m_{K} \rho_{1} h\left(c_{K}^{m}\right) f_{u_{T, K}^{m}}\left(y_{K}\right) \\
& +m_{K} \beta_{1} y_{K}+m_{K} T_{\text {treat }}\left(t_{m+1}, y_{K}\right), \\
& \circ\left(\mathscr{W}_{c}(y)\right)_{K}=\frac{m_{K}}{\delta t}\left(y_{K}-c_{K}^{n}\right)+\sum_{\sigma_{K L} \in \mathscr{E}_{K}} D_{K L}^{(2)} \eta_{K L}^{m+1}\left(p\left(y_{K}\right)-p\left(y_{L}\right)\right) \\
& -m_{K} \alpha_{2} u_{e, K}^{m}+m_{K} \beta_{2} y_{K}+\gamma_{2} u_{K}^{m} y_{K}, \\
& \circ\left(\mathscr{W}_{u_{e}}(y)\right)_{K}=\frac{m_{K}}{\delta t}\left(y_{K}-u_{e, K}^{n}\right)+\sum_{\sigma_{K L} \in \mathscr{E}_{K}} \Lambda_{K L}^{(3)} \tilde{a}_{K L}^{m+1}\left(y_{K}-y_{L}\right) \\
& -\sum_{\sigma_{K L} \in \mathscr{E}_{K}} \Lambda_{K L}^{(3)} \tilde{a}_{K L}^{m+1} \tilde{\mu}_{K L}^{m+1}\left(v_{K}^{m}-v_{L}^{m}\right) \\
& -m_{K} \rho_{3} f_{u_{T, K}^{m}}\left(y_{K}\right)+m_{K} \beta_{3} y_{K} \\
& \circ\left(\mathscr{W}_{v}(y)\right)_{K}=\frac{m_{K}}{\delta t}\left(y_{K}-v_{K}^{n}\right)+\sum_{\sigma_{K L} \in \mathscr{E}_{K}} D_{K L}^{(4)} \bar{\eta}_{K L}^{m+1}\left(p\left(y_{K}\right)-p\left(y_{L}\right)\right) \\
& -m_{K} \alpha_{4} g\left(c_{K}^{m}\right) u_{K}^{m}+m_{K} \beta_{4} y_{K}+\gamma_{4} u_{e, K}^{m} y_{K} .
\end{aligned}
$$

Observe that if $\mathscr{W}\left(u^{m+1}, c^{m+1}, u_{e}^{m+1}, v^{m+1}\right)=0$ then $\left(u^{m+1}, c^{m+1}, u_{e}^{m+1}, v^{m+1}\right)$ is a solution of (29)-(32).

The aim in the first step is to prove that $\exists k \geq 0, \forall\|y\|_{2}>k:(\mathscr{W}(y), y) \geq 0$. So we develop the expression of $(\mathscr{W}(y), y)=\left(\mathscr{W}_{u}\left(y_{u}\right), y_{u}\right)+\left(\mathscr{W}_{c}\left(y_{c}\right), y_{c}\right)+\left(\mathscr{W}_{u_{e}}\left(y_{u_{e}}\right), y_{u_{e}}\right)+$ 
$\left(\mathscr{W}_{v}\left(y_{v}\right), y_{v}\right), y=\left(y_{u}, y_{c}, y_{u_{e}}, y_{v}\right)$ to build inequalities. We have

$$
\begin{aligned}
& \circ\left(\mathscr{W}_{u}(y), y\right)=\sum_{K \in \vartheta} \frac{m_{K}}{\delta t} y_{K}^{2}-\sum_{K \in \vartheta} \frac{m_{K}}{\delta t} u_{K}^{n} y_{K}+\sum_{\sigma_{K L} \in \mathscr{E}} \Lambda_{K L}^{(1)} a_{K L}^{m+1}\left(y_{K}-y_{L}\right)^{2} \\
& -\sum_{\sigma_{K L} \in \mathscr{E}} \Lambda_{K L}^{(1)} a_{K L}^{m+1} \mu_{K L}^{m+1}\left(c_{K}^{m}-c_{L}^{m}\right)\left(y_{K}-y_{L}\right)-\sum_{K \in \vartheta} m_{K} \rho_{1} h\left(c_{K}^{m}\right) f_{u_{T, K}^{m}}\left(y_{K}\right) y_{K} \\
& +\sum_{K \in \vartheta} m_{K} \beta_{1} y_{K}^{2}+\sum_{K \in \vartheta} m_{K} T_{\text {treat }}\left(t_{m+1}, y_{K}\right) y_{K}, \\
& \circ\left(\mathscr{W}_{c}(y), y\right)=\sum_{K \in \vartheta} \frac{m_{K}}{\delta t} y_{K}^{2}-\sum_{K \in \vartheta} \frac{m_{K}}{\delta t} c_{K}^{n} y_{K}+\sum_{\sigma_{K L} \in \mathscr{E}} D_{K L}^{(2)} \eta_{K L}^{m+1}\left(p\left(y_{K}\right)-p\left(y_{L}\right)\right)\left(y_{K}-y_{L}\right) \\
& -\sum_{K \in \vartheta} m_{K} \alpha_{2} u_{e, K}^{m} y_{K}+\sum_{K \in \vartheta} m_{K} \beta_{2} y_{K}^{2}+\sum_{K \in \vartheta} \gamma_{2} u_{K}^{m} y_{K}^{2} \\
& \circ\left(\mathscr{W}_{u_{e}}(y), y\right)=\sum_{K \in \vartheta} \frac{m_{K}}{\delta t} y_{K}^{2}-\sum_{K \in \vartheta} \frac{m_{K}}{\delta t} u_{e, K}^{n} y_{K}+\sum_{\sigma_{K L} \in \mathscr{E}} \Lambda_{K L}^{(3)} \tilde{a}_{K L}^{m+1}\left(y_{K}-y_{L}\right)^{2} \\
& -\sum_{\sigma_{K L} \in \mathscr{E}} \Lambda_{K L}^{(3)} \tilde{a}_{K L}^{m+1} \tilde{\mu}_{K L}^{n+1}\left(v_{K}^{m}-v_{L}^{m}\right)\left(y_{K}-y_{L}\right) \\
& -\sum_{K \in \vartheta} m_{K} \rho_{3} f_{u_{T, K}^{m}}\left(y_{K}\right) y_{K}+\sum_{K \in \vartheta} m_{K} \beta_{3} y_{K}^{2} \\
& \circ\left(\mathscr{W}_{v}(y), y\right)=\sum_{K \in \vartheta} \frac{m_{K}}{\delta t} y_{K}^{2}-\sum_{K \in \vartheta} \frac{m_{K}}{\delta t} v_{K}^{n} y_{K}+\sum_{\sigma_{K L} \in \mathscr{E}} D_{K L}^{(4)} \bar{\eta}_{K L}^{m+1}\left(p\left(y_{K}\right)-p\left(y_{L}\right)\right)\left(y_{K}-y_{L}\right) \\
& -\sum_{K \in \vartheta} m_{K} \alpha_{4} g\left(c_{K}^{m}\right) u_{K}^{m} y_{K}+\sum_{K \in \vartheta} m_{K} \beta_{4} y_{K}^{2}+\sum_{K \in \vartheta} \gamma_{4} u_{e, K}^{m} y_{K}^{2} .
\end{aligned}
$$

With Lemma 1 , we know that $\sum_{\sigma_{K L} \in \mathscr{E}} \Lambda_{K L}^{(1)} a_{K L}^{m+1}\left(y_{K}-y_{L}\right)^{2} \geq 0, \sum_{\sigma_{K L} \in \mathscr{E}} \Lambda_{K L}^{(3)} \tilde{a}_{K L}^{m+1}\left(y_{K}-\right.$ $\left.y_{L}\right)^{2} \geq 0$ and with Lemma 2 we have $\sum_{\sigma_{K L} \in \mathscr{E}} D_{K L}^{(2)} \eta_{K L}^{m+1}\left(p\left(y_{K}\right)-p\left(y_{L}\right)\right)\left(y_{K}-y_{L}\right) \geq 0$, $\sum_{\sigma_{K L} \in \mathscr{E}} D_{K L}^{(4)} \bar{\eta}_{K L}^{m+1}\left(p\left(y_{K}\right)-p\left(y_{L}\right)\right)\left(y_{K}-y_{L}\right) \geq 0$ because $p(\cdot)$ is non decreasing on $\mathbb{R}$. Then we have $\forall \lambda>0$

$$
\begin{aligned}
&-\sum_{\sigma_{K L} \in \mathscr{E}} \Lambda_{K L}^{(1)} a_{K L}^{m+1} \mu_{K L}^{m+1}\left(c_{K}^{m}-c_{L}^{m}\right)\left(y_{K}-y_{L}\right) \\
& \geq-\frac{\lambda^{2}}{2} \sum_{\sigma_{K L} \in \mathscr{E}}\left(\Lambda_{K L} a_{K L}^{m+1} \mu_{K L}^{m+1}\left(c_{K}^{m}-c_{L}^{m}\right)\right)^{2}-\frac{1}{2 \lambda^{2}} \sum_{\sigma_{K L} \in \mathscr{E}}\left(y_{K}-y_{L}\right)^{2} \\
& \quad-\frac{\lambda^{2}}{2} \sum_{\sigma_{K L} \in \mathscr{E}}\left(\Lambda_{K L} a_{L^{\infty}(\mathbb{R})} \mu_{L^{\infty}(\mathbb{R})}\left(c_{K}^{m}-c_{L}^{m}\right)\right)^{2}-\frac{2}{\lambda^{2}}(\# \mathscr{E})\|y\|_{2}^{2},
\end{aligned}
$$

and

$$
\begin{aligned}
& -\sum_{\sigma_{K L} \in \mathscr{E}} \Lambda_{K L}^{(3)} \tilde{a}_{K L}^{m+1} \tilde{\mu}_{K L}^{m+1}\left(v_{K}^{m}-v_{L}^{m}\right)\left(y_{K}-y_{L}\right) \\
& \geq-\frac{\lambda^{2}}{2} \sum_{\sigma_{K L} \in \mathscr{E}}\left(\Lambda_{K L}^{(3)} \tilde{a}_{K L}^{m+1} \tilde{\mu}_{K L}^{m+1}\left(v_{K}^{m}-v_{L}^{m}\right)\right)^{2}-\frac{1}{2 \lambda^{2}} \sum_{\sigma_{K L} \in \mathscr{E}}\left(y_{K}-y_{L}\right)^{2} \\
& \quad \geq-\frac{\lambda^{2}}{2} \sum_{\sigma_{K L} \in \mathscr{E}}\left(\Lambda_{K L}^{(3)} \tilde{a}_{L^{\infty}(\mathbb{R})} \tilde{\mu}_{L^{\infty}(\mathbb{R})}\left(v_{K}^{m}-v_{L}^{m}\right)\right)^{2}-\frac{2}{\lambda^{2}}(\# \mathscr{E})\|y\|_{2}^{2}
\end{aligned}
$$


So, we have those inequalities

$$
\begin{aligned}
& \left(\mathscr{W}_{u}\left(y_{u}\right), y_{u}\right) \geq C_{1}^{(u)}\left\|y_{u}\right\|_{2}^{2}-C_{2}^{(u)}\left\|y_{u}\right\|_{2}-C_{3}^{(u)}, \\
& \left(\mathscr{W}_{c}\left(y_{c}\right), y_{c}\right) \geq C_{1}^{(c)}\left\|y_{c}\right\|_{2}^{2}-C_{2}^{(c)}\left\|y_{c}\right\|_{2}, \\
& \left(\mathscr{W}_{u_{e}}\left(y_{u_{e}}\right), y_{u_{e}}\right) \geq C_{1}^{\left(u_{e}\right)}\left\|y_{u_{e}}\right\|_{2}^{2}-C_{2}^{\left(u_{e}\right)}\left\|y_{u_{e}}\right\|_{2}-C_{3}^{\left(u_{e}\right)}, \\
& \left(\mathscr{W}_{v}\left(y_{v}\right), y_{v}\right) \geq C_{1}^{(v)}\left\|y_{v}\right\|_{2}^{2}-C_{2}^{(v)}\left\|y_{v}\right\|_{2},
\end{aligned}
$$

with the following expression for the constants, choosing $\lambda$ such that $\lambda^{2}>\frac{2(\# \mathscr{E}) \delta t}{m_{\mathscr{M}}^{\text {down }}}$ and denoting $m_{\mathscr{M}}^{\text {down }}=\min _{K \in \vartheta} m_{K} \leq m_{K} \leq \max _{K \in \vartheta} m_{K}=m_{\mathscr{M}}^{u p}$

$$
\begin{aligned}
& C_{1}^{(u)}=\frac{m_{\mathscr{M}}^{\text {down }}}{\delta t}-\frac{2(\# \mathscr{E})}{\lambda^{2}}>0, \quad C_{2}^{(u)}=\frac{m_{\mathscr{M}}^{u p}}{\delta_{t}}(\# \vartheta)+m_{\mathscr{M}}^{u p} \rho_{1} f_{L^{\infty}(\mathbb{R})}(\# \vartheta)>0, \\
& C_{3}^{(u)}=\frac{\lambda^{2}}{2} \sum_{\sigma_{K L} \in \mathscr{E}}\left(\Lambda_{K L}^{(1)} a_{L^{\infty}(\mathbb{R})} \mu_{L^{\infty}(\mathbb{R})}\left(c_{K}^{m}-c_{L}^{m}\right)\right)^{2}>0, \\
& C_{1}^{(c)}=\frac{m_{\mathscr{M}}^{\text {down }}}{\delta t}>0, \quad C_{2}^{(c)}=m_{\mathscr{M}}^{u p}(\# \vartheta)\left(\frac{\max _{K \in}\left(c_{K}^{m}\right)}{\delta t}+\alpha_{2}\right)>0, \\
& C_{1}^{\left(u_{e}\right)}=\frac{m_{\mathscr{M}}^{\text {down }}}{\delta t}-\frac{2(\# \mathscr{E})}{\lambda^{2}}>0, \quad C_{2}^{\left(u_{e}\right)}=\frac{m_{\mathscr{M}}^{u p}(\# \vartheta)+m_{\mathscr{M}}^{u p} \rho_{3} f_{L^{\infty}(\mathbb{R})}(\# \vartheta)>0,}{\delta_{t}}(\#) \\
& C_{3}^{\left(u_{e}\right)}=\frac{\lambda^{2}}{2} \sum_{\sigma_{K L} \in \mathscr{E}}\left(\Lambda_{K L}^{(3)} \tilde{a}_{L^{\infty}(\mathbb{R})} \tilde{\mu}_{L^{\infty}(\mathbb{R})}\left(v_{K}^{m}-v_{L}^{m}\right)\right)^{2}>0, \\
& C_{1}^{(v)}=\frac{m_{\mathscr{M} n}^{d o w n}}{\delta t}>0, \quad C_{2}^{(v)}=m_{\mathscr{M}}^{u p}(\# \vartheta)\left(\frac{\max _{K \in \vartheta}\left(v_{K}^{m}\right)}{\delta t}+\alpha_{4}\right)>0 .
\end{aligned}
$$

It induces that

$$
\begin{aligned}
(\mathscr{W}(y), y) \geq \min \left(C_{1}^{(u)}, C_{1}^{(c)}, C_{1}^{\left(u_{e}\right)},\right. & \left.C_{1}^{(v)}\right)\|y\|_{2}^{2} \\
& \quad-\max \left(C_{2}^{(u)}, C_{2}^{(c)}, C_{2}^{\left(u_{e}\right)}, C_{2}^{(v)}\right)\|y\|_{2}-C_{3}^{(u)}-C_{3}^{\left(u_{e}\right)} .
\end{aligned}
$$

So there exists $k>0$ from which $\forall\|y\|_{2}^{2} \geq k:(\mathscr{W}(y), y)>0$.

Suppose that there is no $z \in \mathbb{R}^{4 \times \# \vartheta}: \mathscr{W}(z)=0$, in that case we can define the application $\mathscr{S}: y \in \mathscr{B}(0, k) \mapsto-k \frac{\mathscr{W}(y)}{\|\mathscr{W}(y)\|} \in \mathscr{B}(0, k) . \mathscr{S}$ is continuous due to $\mathscr{W}$, so according to the Brouwer fixed point theorem there exists a fixed point $\tilde{y}$ of $\mathscr{S}$ on $\mathscr{B}(0, k)$ :

$$
\tilde{y}=-k \frac{\mathscr{W}(\tilde{y})}{\|\mathscr{W}(\tilde{y})\|}
$$

Taking the norm of $\tilde{y}$ from (46), we get $\|\tilde{y}\|=k>0$ but taking the inner product of (46) with $\tilde{y}$ we get : $\|\tilde{y}\|^{2}=-k \frac{(\mathscr{W}(\tilde{y}), \tilde{y})}{\|\mathscr{W}(\tilde{y})\|} \leq 0$. Thus there exists $z$ with $\mathscr{W}(z)=0$. So there exists a solution $\left(u^{m+1}, c^{m+1}, u_{e}^{m+1}, v^{m+1}\right)$ to (29)-(32). 


\section{Modeling Treatments}

Surgery, chemotherapy and radiotherapy are the treatments commonly used against GBM, all of them can be modeled in (1a)-(1d). Surgery is performed in emergency as soon as GBM is diagnosed. However some patients can not undergo surgery due to the non-accessibility of the tumour, in that case only a biopsy is done. If surgery is performed, the tumour core is removed but resection goes the largest possible without damaging healthy tissues.

In our model, we suppose that surgery is performed at a time-step $t_{\text {surg }}$ that can be either the initial time $t_{0}$ or a random time-step $t_{n}, n \in \llbracket 1, N+1 \rrbracket$. If $t_{\text {surg }}=t_{n}, n \in$ $\llbracket 1, N+1 \rrbracket$ then $\forall m \in \llbracket 1, n-1 \rrbracket, u^{m}$ is calculated according to (12)-(15) and $\forall K \in$ $\vartheta, w=u, c, u_{e}, v$

$$
w\left(t_{\text {surg }}, x_{K}\right)=\left\{\begin{array}{l}
w_{K}^{n-1}, \text { if } x_{K} \notin \text { surgical area } \\
0, \text { if } x_{K} \in \text { surgical area }
\end{array}\right.
$$

Finally $\forall m \in \llbracket n+1, N+1 \rrbracket, u^{m}$ is calculated according to (12)-(15) with the new initial conditions $w_{0}=w\left(t_{\text {surf }}, \cdot\right), w=u, c, u_{e}, v$.

If $t_{\text {surg }}=t_{0}$ then we solve (12)-(15) with the new initial conditions $w_{0}^{\text {surg }}, w=u, c, u_{e}, v$

$$
w_{0}^{\text {surg }}=\left\{\begin{array}{l}
w_{0}, \text { if } x_{K} \notin \text { surgical area }, \\
0, \text { if } x_{K} \in \text { surgical area } .
\end{array}\right.
$$

Chemotherapy is the use of a drug designed against a tumour cell population. The drug commonly used in GBM is TMZ at a daily dose of $75 \mathrm{mg} / \mathrm{m}^{2}$ (Stupp et al, 2005). The chemotherapy part of treatments is modeled by

$$
T_{\text {chemo }}(t, u)=k_{c}(t) D_{\text {che }} u,
$$

where $k_{c}(t)=1$ if and only if the chemotherapy is effective at the time $\mathrm{t}$ and $D_{\text {che }}$ is the dose administered by the drug.

The problem of chemotherapy is the possible existence of tumour-resistant cells that are not affected by the drug. Those cells can still proliferate in the tumour site causing relapses for the patient. For this work, we do not consider these sub-type of cancer cells and suppose that all tumour cells are affected by the drug. However, we know that the use of chemotherapy enhances the performances of radiotherapy because the drug makes the tumour cells more sensitive to radiations (Stupp et al, 2005).

Radiotherapy occurs for almost all cancer treatments because the use of radiotherapy depends mainly on the location of the tumour and its spatial spread and not on the cancer type. Indeed radiotherapy works by sending a dose of radiations at a local position (the tumour location), those radiations cause micro-breaks into the DNA of irradiated cells, normal cells can repair those DNA breaks but tumour cells often can not causing their death. Radiotherapies can cause side effects if normal cells are altered by the radiations, explaining why each cancer type has a specific guideline to dose the quantity of radiations allowed.

With GBM, radiotherapy is done by administering the dose of radiations in small fractions. The number of fractions and the cumulative dose depend on the patient 
health, for example its age or its WHO performance status (ANOCEF, 2018). The efficiency of radiotherapy depends on a lot of parameters: the number of fractions per day $N_{\text {frac }}$, the dose administered $D_{\text {rad }}$, the duration of irradiation $\tau$, the time between irradiation $\Delta \tau$, the DNA damaged rate $\mu$ and sensitivity parameters $\alpha, \beta$. In France, it is recommended to perform radiotherapy 5 days a week for 6 weeks with 30 fractions of $2 G_{\gamma}$ per day(ANOCEF, 2018).

Because, multiple small fractions are delivered with GBM, we model radiotherapy based on (Nilsson et al, 1990)

$$
T_{\text {radio }}(t, u)=k_{\text {chemo }}(t) k_{r}(t) R_{e f f} u \mathbb{1}_{\{x \in \text { irradiated area }\}}(x),
$$

where $k_{r}(t)=1$ if and only if the radiotherapy is effective at the time $\mathrm{t}$ and

$$
\begin{gathered}
R_{e f f}=\alpha N_{f r a c} D_{r a d}+\beta N_{\text {frac }} D_{r a d}^{2}\left(g_{\text {rad }}(\mu \tau)+2\left(\frac{\cosh (\mu \tau)-1}{(\mu \tau)^{2}}\right) h_{N_{\text {frac }}}(\varphi)\right), \\
g_{\text {rad }}(\mu \tau)=2\left(\frac{\mu \tau-1+\exp (-\mu \tau)}{(\mu \tau)^{2}}\right), \\
\varphi=\exp (-\mu(\tau+\Delta \tau)), \\
h_{m}(\varphi)=2\left(\frac{m \varphi-m \varphi^{2}-\varphi+\varphi^{m+1}}{m(1-\varphi)^{2}}\right) .
\end{gathered}
$$

We use the term $k_{\text {chemo }}(t)$ to model the enhance efficiency of radiotherapy if chemotherapy is applied concomitant with radiotherapy. So if chemotherapy is not administered at the time $t$, we set $k_{\text {chemo }}(t)$ to 1 and if radiotherapy and chemotherapy are concomitant then $k_{\text {chemo }}(t)=\Upsilon>1, \Upsilon$ is the efficiency rate improvement of using radiotherapy with chemotherapy.

\section{Methods}

We use MRIs from the patient C3L_16 in the CPTAC-GBM database (TCIA, 2018). This patient is a 60 year-old male (BMI of 28.81 and BSA of $2.07 \mathrm{~m}^{2}$ ) diagnosed with a Glioblastoma of $4.3 \mathrm{~cm}$ in his parietal lobe who died of Glioblastoma 77 days after diagnosis.

There are different types of MRI that can be used to extract information like shown in figure 2: a T1 highlights the white matter in white while grey matter is not highlighted, a T1-Gado separates well the tumour core and edema from the brain, T2 and FLAIR show the enhancing tumour area.

From those MRI, we build the triangular mesh $\mathscr{T}$ on which our model takes place. We use the software AutoCAD (version O.161.0.0 AutoCAD 2018.1.2 Update) to place, manually, vertex on the outside of the brain, of the tumour core, of the edema and of the enhancing tumour. We attribute edges to those vertex and use the software Triangle (version 1.6) (J.R., 1996; Shewchuk, 2002) to get the triangulation $\mathscr{T}$. The primal and dual mesh obtained are represented in the figure $3 \mathrm{a}$. Parameters of the primal mesh $\mathscr{T}$ are given in table 1 . With the information readable in the MRIs of the figure 2, we can extract manually data in the brain: the white/grey matter locations 


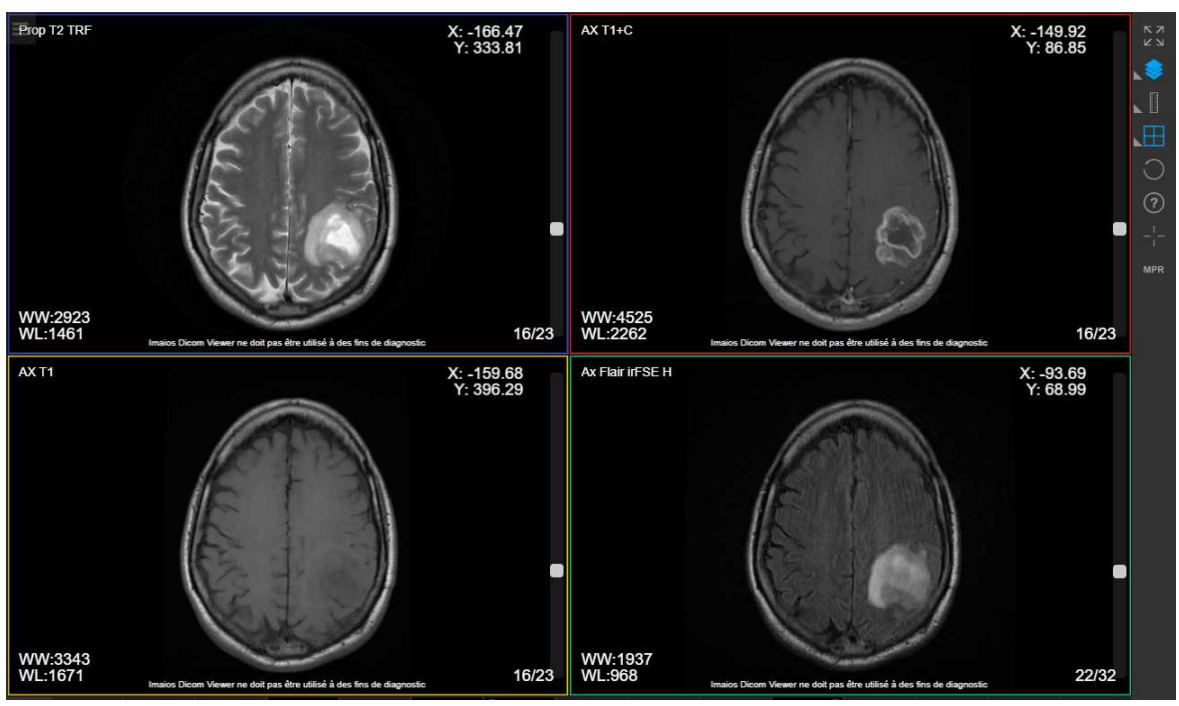

Fig. 2: Extract of four axial MRIs from the patient C3L_16 in (TCIA, 2018). Those MRIs are the four basis MRI sequences required for Glioblastoma diagnosis. Those sequences are a T2 on the top left, a T1 weighted (or T1 with gadolinium) on the top right, a T1 on the bottom left and a T2 weighted (or FLAIR) on the bottom right.

and the tumour segmentation. The segmentation of the brain is represented in the figure $3 \mathrm{~b}$ in which we have in different shades of blue, the locations of grey matter (in dark blue) and the white matter (in light blue) and the whole tumour which is divided into three parts: the tumour core with hypoxic cells in green, the edema in yellow and the enhancing tumour in red. We suppose before surgery that the diffusion in the whole tumour behaves like the white matter.

To solve the numerical scheme (12)-(15) we follow the same guidelines than the discrete properties. From each time step $t_{n}, n \geq 0$ we use the number sequence $X_{m+1}=$ $\tau^{(n)}\left(X_{m}\right)$ with the semi-implicit numerical scheme (29)-(32).

We consider that $X_{m+1}=\left(u^{n+1}, c^{n+1}, u_{e}^{n+1}, v^{n+1}\right)$ when the relative error follows

$$
\frac{\left\|w^{m+1}-w^{m}\right\|_{2}}{\left\|w^{m}\right\|_{2}}<\text { tolImplicit, with } w=u, c, u_{e}, v
$$

with tolImplicit $=10^{-3}$ being a numerical threshold. To find $X_{m+1}$ from $X_{m}$ we solve (29)-(32) using Newton's method with $10^{-11}$ as the error of convergence. Observe that choosing to solve the semi-implicit scheme (29)-(32) instead of the coupled system (12)-(15) allows to solve four smaller systems rather than one system four times wider.

Finally we use a conjugate gradient method ending with a numerical threshold tolGradient of $10^{-13}$ to solve each step of the Newton method. 


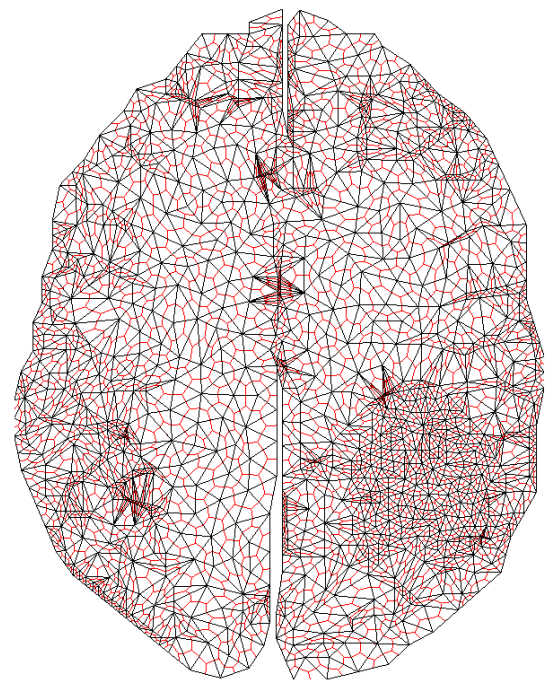

(a) primal and dual mesh

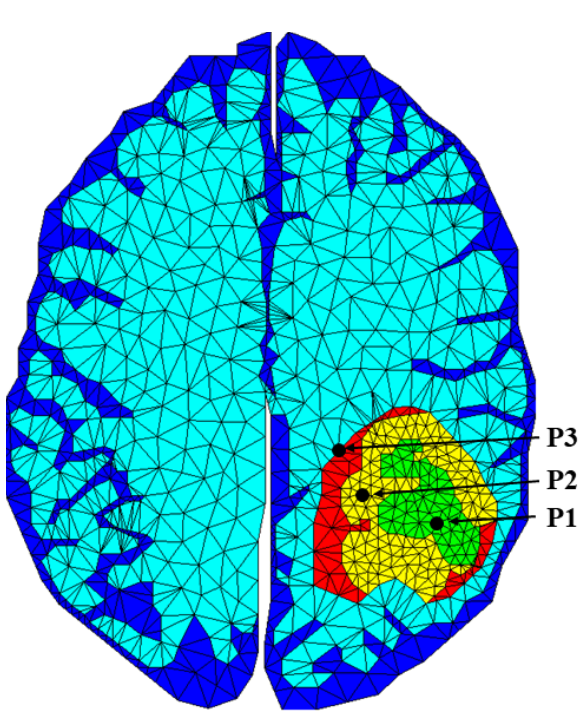

(b) segmentation of the brain

Fig. 3: (a) Primal mesh $\mathscr{T}$ (in black) and dual mesh $\mathscr{M}$ (in red) corresponding to the MRIs in figure2. (b) Segmentation of the brain on the primal mesh $\mathscr{T}$ according to figure 2: the grey matter in dark blue, the white matter in light blue, the tumour core in green, the edema in yellow and the enhancing tumour in red. $P 1, P 2$ and $P 3$ are three points used for investigating the tumor cell concentration in the area of the tumour.

Table 1: Parameters from the primal and dual meshes including the number of vertex, triangles, edges, interior vertex, the minimum and the maximum area on $\mathscr{M}$.

\begin{tabular}{cccccc}
\hline vertex & triangles & edges & interior vertex & $\min _{K \in \vartheta} m_{K}$ & $\max _{K \in \vartheta} m_{K}$ \\
\hline 1083 & 1963 & 3022 & 423 & $6.47 \times 10^{-6}$ & $1.91 \times 10^{-4}$ \\
\hline
\end{tabular}

\section{Numerical simulations}

We choose to model the diffusion and chemotaxis with the following functions according to assumptions (A1) and (A2)

$$
\begin{aligned}
& a(y)=y(1-y) \mathbb{1}_{[0,1]}(y), \\
& \chi_{1}(y)=\lambda_{1}(y(1-y))^{2} \mathbb{1}_{[0,1]}(y), \lambda_{1}=1.0 \mathrm{~cm}^{2} \cdot \text { day }^{-1} \cdot \mu m o l^{-1}(\text { Anderson, 2005), } \\
& \chi_{3}(y)=\lambda_{3}(y(1-y))^{2} \mathbb{1}_{[0,1]}(y), \lambda_{3}=2.25 \times 10^{4} \mathrm{~cm}^{2} \cdot d a y^{-1} \cdot \mu \mathrm{mol}^{-1} .
\end{aligned}
$$

Even though a more detailed anisotropic expression has been developed for the diffusion in the white matter (K.J.Paintera and T.Hillen, 2013), we choose here an easier 
Table 2: Values of the coefficients used in (1a)-(1d) common in all numerical simulations

\begin{tabular}{|c|c|c|c|c|}
\hline Coefficients & Values & Units & Descriptions & References \\
\hline$\rho_{1}$ & $2.7 \times 10^{-1}$ & $d a y^{-1}$ & tumour cell growth rate & (Curtin et al, 2020) \\
\hline$\beta_{1}$ & $1.7 \times 10^{-1}$ & $d a y^{-1}$ & tumour cell apoptosis rate & $\begin{array}{l}\text { (Lai and Friedman, } \\
\text { 2020) }\end{array}$ \\
\hline$\alpha_{2}$ & 100 & $\mu m o l \cdot d a y^{-1}$ & $\begin{array}{l}\mathrm{O}_{2} \text { production rate by en- } \\
\text { dothelial cells }\end{array}$ & this work \\
\hline$\beta_{2}$ & $3.75 \times 10^{-2}$ & $d a y^{-1}$ & $\mathrm{O}_{2}$ degradation rate & (Anderson, 2005) \\
\hline$\gamma_{2}$ & 6 & $d a y^{-1}$ & $\begin{array}{l}\mathrm{O}_{2} \text { consumption rate by } \\
\text { tumour cells }\end{array}$ & this work \\
\hline$\rho_{3}$ & $4.9 \times 10^{-3}$ & $d a y^{-1}$ & $\begin{array}{l}\text { endothelial cell growth } \\
\text { rate }\end{array}$ & this work \\
\hline$\beta_{3}$ & $3.1 \times 10^{-3}$ & $d a y^{-1}$ & $\begin{array}{l}\text { endothelial cell apoptosis } \\
\text { rate }\end{array}$ & this work \\
\hline$\alpha_{4}$ & 340 & $\mu \mathrm{mol} \cdot d a y^{-1}$ & $\begin{array}{l}\text { VEGF production rate by } \\
\text { tumour cells }\end{array}$ & this work \\
\hline$\beta_{4}$ & 15.6 & $d a y^{-1}$ & VEGF degradation rate & (Curtin et al, 2020) \\
\hline$\gamma_{4}$ & 1.4 & $d a y^{-1}$ & $\begin{array}{l}\text { VEGF consumption by } \\
\text { endothelial cells }\end{array}$ & (Curtin et al, 2020) \\
\hline$u_{\max }$ & $2.39 \times 10^{8}$ & cells $\cdot \mathrm{cm}^{-2}$ & maximum tissue capacity & (Curtin et al, 2020) \\
\hline$d_{g m}$ & $2.7 \times 10^{-4}$ & $c m^{2} \cdot d a y^{-1}$ & $\begin{array}{l}\text { diffusion rate of cells in } \\
\text { grey matter }\end{array}$ & (Curtin et al, 2020) \\
\hline$d_{w m}$ & $5 d_{g m}$ & $c m^{2} \cdot d a y^{-1}$ & $\begin{array}{l}\text { diffusion rate of cells in } \\
\text { white matter }\end{array}$ & (Jbabdi et al, 2005) \\
\hline$d_{p s}$ & $50 d_{g m}$ & $\mathrm{~cm}^{2} \cdot d a y^{-1}$ & $\begin{array}{l}\text { diffusion rate of cells in } \\
\text { the post-surgical area }\end{array}$ & this work \\
\hline$d_{2}$ & 8.6 & $c m^{2} \cdot d a y^{-1}$ & diffusion rate of $\mathrm{O}_{2}$ & this work \\
\hline$d_{4}$ & $8.6 \times 10^{-1}$ & $c m^{2} \cdot d a y^{-1}$ & diffusion rate of VEGF & this work \\
\hline$c_{\text {hypo }}$ & 75 & $\mu \mathrm{mol} \cdot \mathrm{cm}^{-2}$ & $\begin{array}{l}\text { threshold under which } \\
\text { cells are in hypoxia }\end{array}$ & this work \\
\hline$c_{\text {necro }}$ & 50 & $\mu \mathrm{mol} \cdot \mathrm{cm}^{-2}$ & $\begin{array}{l}\text { threshold under which } \\
\text { cells necrose }\end{array}$ & this work \\
\hline
\end{tabular}

expression in order to don't use Diffusion Tensor Imaging (DTI) data. The diffusion in the white matter is then set to be 5 times faster than in the grey matter, and in the post-surgical area, we set the diffusion to be 50 times faster than in the grey matter:

$$
\begin{aligned}
& \Lambda_{1}(x)=d_{1}(x)\left[\begin{array}{ll}
1 & 0 \\
0 & 1
\end{array}\right], \text { with } d_{1}(x) \begin{cases}d_{w m} & \text { if } x \text { is in the white matter, } \\
d_{g m} & \text { if } x \text { is in the grey matter, } \\
d_{p s} & \text { if } x \text { is in the post-surgical area, }\end{cases} \\
& D_{2}=d_{2}\left[\begin{array}{ll}
1 & 0 \\
0 & 1
\end{array}\right], \\
& \Lambda_{3}(x)=1.8 \times 10^{-2} \times \Lambda_{1}(x) \text { as proposed in (Curtinet al, 2020), }
\end{aligned}
$$




$$
D_{4}=d_{4}\left[\begin{array}{ll}
1 & 0 \\
0 & 1
\end{array}\right] \text {. }
$$

The different coefficients in (1a)-(1d) following the assumption (A4) are given in table 2.

Following the timeline of a patient with GBM (ANOCEF, 2018), we set the treatments as followed:

- Surgery is performed on the first day of simulation, that surgery removes every cells and proteins in the necrotic, enhancing and whole tumour area as segmented in the figure $3 \mathrm{~b}$.

- Radiotherapy and chemotherapy start both on the $14^{\text {th }}$ day and last for a period of 6 weeks.

- Chemotherapy is administered everyday of the treatment schedule, so $k_{c}(t)=$ $\mathbb{1}_{[14,56]}(t)$ and $k_{\text {chemo }}(t)=\Upsilon \cdot \mathbb{1}_{[14,56]}(t)$.

- Radiotherapy is administered periodically 5 days in a row with 2 days off, so $k_{r}(t)=\mathbb{1}_{[14,19] \cup[21,26] \cup[28,33] \cup[35,40] \cup[42,47] \cup[49,54]}(t)$.

- Radiotherapy is administered locally where the surgery was performed and also in the $3 \mathrm{~cm}$ area bordering the whole tumour area. This bordering area is computed manually.

Experiments in (Stupp et al, 2005) found a 33\% increase in median survival comparing radiotherapy only and radiotherapy with TMZ but there is no study using chemotherapy only on patients. $\Upsilon$ is chosen to be $\frac{4}{3}$ even though the value is overestimated.

The value of all parameters related to treatments are summarized in table 3. Most of them come from the work of (Powathil et al, 2007) with the current state of treatments in (ANOCEF, 2018).

Six simulations have been done to solve (1a)-(3), depending on the treatments administered to the patient: with or without surgery, chemotherapy and radiotherapy only (experiments 1 to 4 ) and then the concomitant use of chemotherapy and radiotherapy with or without surgery (experiments 5 and 6). The simulations' settings are given in table 4 using the coefficients from table 2 and table 3.

For the initial conditions, we set the values of $u_{0}$ and $u_{e_{0}}$ from the MRIs of the patient given in figure 2 . According to the location on the MRIs of the tumour core and edema, we set $u_{0}$ to $12 \times 10^{4}$ cells $\cdot \mathrm{cm}^{-2}$ in the tumour core and to $5.5 \times 10^{7}$ cells . $\mathrm{cm}^{-2}$ in the edema. In the enhancing tumour area, we set the tumour cells population with $u_{0}(x)=1.4 \times 10^{8} \exp \left(-\frac{4}{45}\left|x-x_{c}\right|\right)$ cells $\cdot \mathrm{cm}^{-2}$, where $x_{c}$ represents the position of the tumour's center, and outside of those areas we set $u_{0}$ to 0 . According to the location of the grey and white matter, we set $u_{e_{0}}$ to be $7.17 \times 10^{7}$ cells $\cdot \mathrm{cm}^{-2}$ in the grey matter and to $2.39 \times 10^{5}$ cells $\cdot \mathrm{cm}^{-2}$ in the white matter. The initial spatial distribution of tumour and endothelial cells are shown in the figure 4 .

As $\mathrm{O}_{2}$ and VEGF concentrations cannot be seen on MRIs required for Glioblastoma diagnosis, such as those presented in the figure 2, we have attributed their initial concentrations by solving 13 and 15 using the previous expressions of $u_{0}$ and $u_{e_{0}}$ but without the partial temporal term. The computed initial concentration in $\mathrm{O}_{2}$ and VEGF are shown in the figure 5, they are not perfectly fitted with their expected 
Table 3: Parameters around the treatments

\begin{tabular}{|c|c|c|c|c|}
\hline Parameters & Values & Units & Descriptions & References \\
\hline$\alpha$ & $\frac{2.7}{30} \times 10^{-2}$ & $G_{\gamma}^{-1}$ & sensitivity parameter & $\begin{array}{l}\text { (Powathil et al, 2007; } \\
\text { ANOCEF, 2018) }\end{array}$ \\
\hline$\beta$ & $\frac{2.7}{30} \times 10^{-3}$ & $G_{\gamma}^{-2}$ & sensitivity parameter & $\begin{array}{l}\text { (Powathil et al, 2007; } \\
\text { ANOCEF, 2018) }\end{array}$ \\
\hline$N_{\text {frac }}$ & 30 & & $\begin{array}{l}\text { number of fractions per } \\
\text { day }\end{array}$ & (ANOCEF, 2018) \\
\hline$\tau$ & $5.8 \times 10^{-5}$ & $d a y^{-1}$ & duration of irradiation & this work \\
\hline$\Delta \tau$ & $2.9 \times 10^{-4}$ & $d a y^{-1}$ & time between irradiations & this work \\
\hline$\mu$ & 11.04 & $d a y^{-1}$ & DNA damage rate & (Powathil et al, 2007) \\
\hline$r$ & $\frac{4}{3}$ & & $\begin{array}{l}\text { efficiency rate of radio- } \\
\text { therapy with } T M Z\end{array}$ & (Stupp et al, 2005) \\
\hline$D_{\text {che }}$ & $1.96 \times 10^{-2}$ & $d a y^{-1}$ & $\begin{array}{l}\text { death rate of tumour cells } \\
\text { due to chemotherapy }\end{array}$ & (Powathil et al, 2007) \\
\hline$D_{\text {rad }}$ & 2 & $G_{\gamma}$ & $\begin{array}{l}\text { dose administered per ra- } \\
\text { diation }\end{array}$ & (ANOCEF, 2018) \\
\hline
\end{tabular}

Table 4: Recap on the treatments used in the simulations

\begin{tabular}{cccc}
\hline Simulations & Surgery & Chemotherapy (TMZ) & Radiotherapy \\
\hline 1 & no & no & no \\
2 & yes & no & no \\
3 & no & yes & no \\
4 & no & no & yes \\
5 & no & yes & yes \\
6 & yes & yes & yes \\
\hline
\end{tabular}

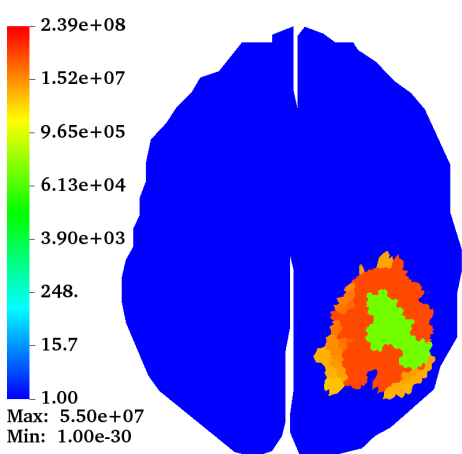

(a) tumour cells

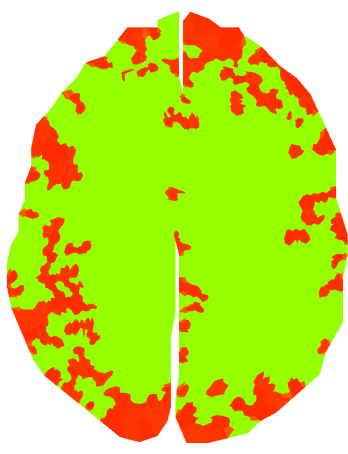

(b) endothelial cells

Fig. 4: (a) The initial number of tumour cells per $\mathrm{cm}^{2}\left(u_{0}\right)$. (b) The initial number of endothelial cells per $\mathrm{cm}^{2}\left(u_{e_{0}}\right)$ on the right. 


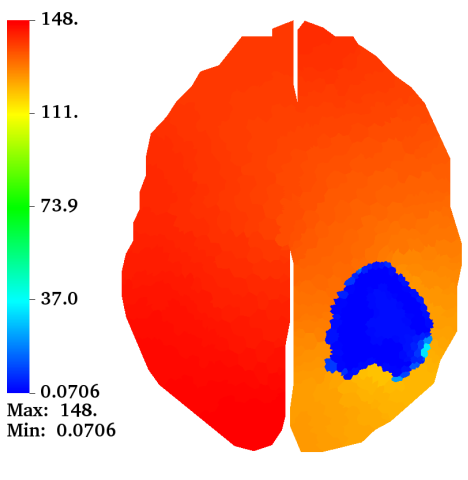

(a) $\mathrm{O}_{2}$ concentration

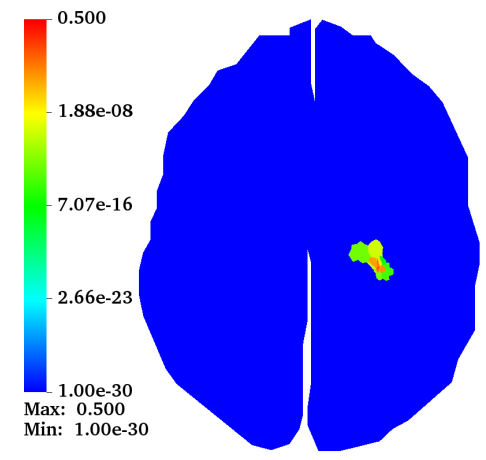

(b) VEGF concentration

Fig. 5: (a) The initial concentration of $O_{2}$ in $\mu \mathrm{mol} . \mathrm{cm}^{-2}\left(c_{0}\right)$. The initial concentration of $V E G F$ in $\mu \mathrm{mol} . \mathrm{cm}^{-2}\left(v_{0}\right)$ on the right.

spatial distributions, which would be smoother around the tumour area, but their behaviour is welly done during the first steps of the simulation.

Indeed, during the simulations, the $\mathrm{O}_{2}$ concentration oscillates asymptotically between 74 and $104 \mu \mathrm{mol} \cdot \mathrm{cm}^{-2}$ and VEGF is produced punctually by the hypoxic tumour cells. Without any treatment the tumour keeps growing inside the brain, inducing the growth of the hypoxic tumour core, the edema and the enhancing tumour area. Moreover without treatment new endothelial cells are produced to supply the growing hypoxic tumour core, who is lacking of $\mathrm{O}_{2}$ to keep proliferating. In order to follow up the impact of treatments on the tumour behaviour, we have displayed in figure 6 the total number of tumour cells through time. We also display in figures 7,8 and 9 the number of tumour cells per $\mathrm{cm}^{2}$ at three points in the brain, namely at $P 1, P 2$ and $P 3 . P 1$ is located in the tumour core, $P 2$ in the edema and $P 3$ in the enhancing tumour area as shown in the figure $3 \mathrm{~b}$. You can find a video of the 6 simulations related in table 4 following this link https : //www . youtube. com/watch?v=vJkMJ5bNoWA.

Depending on the treatments, the tumour growth exhibits different behaviour. Indeed using surgery (simulation 2 and 6) on day 1 allows to decrease intensely the number of tumour cells in the brain, it remains only tumour cells in areas where no evidence of existence were detectable on the different MRIs. However removing the majority of tumour cells does not stop the growth of the tumour because of the cells that have not been affected by the surgery, and no induced angiogenesis is required by them to keep growing because the brain does not lack of $\mathrm{O}_{2}$ anymore. This is why in figure 6 the trajectory of the surgery only curve starts with a big drop on day 1 but comes back to the no treatment curve later on. Surgery is not enough to stop the spread of the tumour growth but it stops locally the spread as there is no tumour cell remaining at $P 1$ and $P 2$ after the surgery, which is why in the figure 7 and 8 the curves where surgery was used are not displayed. $P 3$ is in the surgical area too but also on the boundary of the enhancing tumour area, so some tumour cells near $P 3$ remain after 


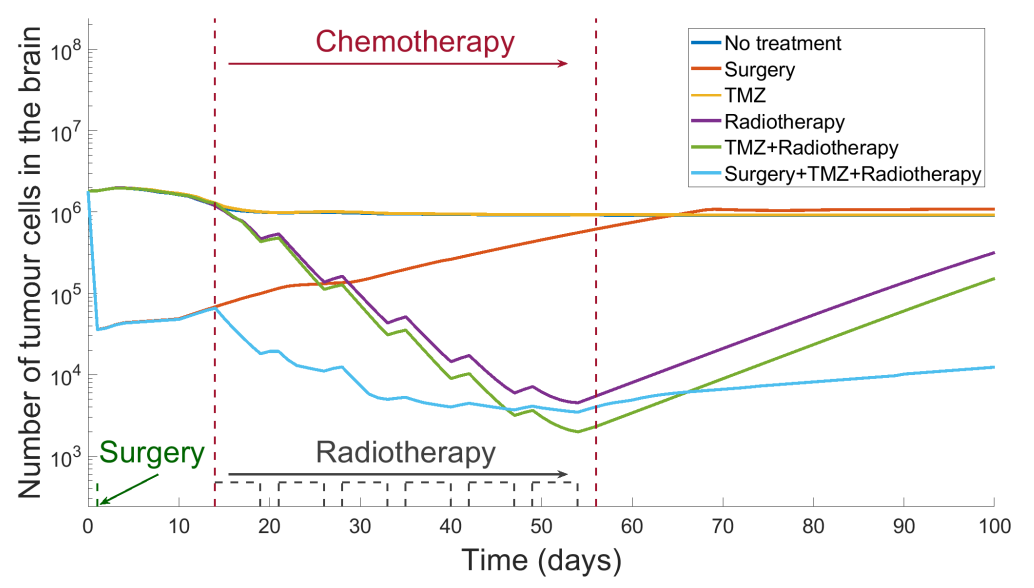

Fig. 6: Number of the total tumour cells in the brain through time for different treatment schedules. If performed, surgery is done on the first day, chemotherapy (TMZ) is administered from day 14 to day 56 and radiotherapy is administered 5 days out of 7 from day 14 to day 54. The curves "No treatment" and "TMZ" are really close and difficult to distinguish at this scale.

the surgery which explains why in the figure 9, there are still tumour cells located at $P 3$ after the surgery.

The use of chemotherapy only with TMZ does not affect the global behaviour of the tumour growth due to the low death rate of tumour cell induced by chemotherapy. In the figure 6 , the trajectory of the chemotherapy only curve is almost perfectly identical to the one with no treatment. Locally the use of TMZ only decreases the number of tumour cells, but that decrease shrinks the hypoxic tumour core area, which means that more tumour cells can replicate themselves than before. Depending on their location, tumour cells can either be in a slightly higher number than without any treatment as at $P 2$ or in a slightly fewer number as at $P 1$ and $P 3$. Those observations explain why no treatment on patients with Glioblastoma rely only on chemotherapy, as this treatment is not sufficient to cure Glioblastoma.

However radiotherapy affects a lot the behaviour of the tumour growth, its use decreases drastically the number of tumour cells in the irradiated area as shown in the figure 6-9. The death rate of tumour cells due to radiotherapy are also enhanced when combined with the chemotherapy as it was exhibited in (Stupp et al, 2005). The efficiency of radiotherapy explains why it is always used, to treat Glioblastoma. In our model, we can see in the figure 6 that the use of radiotherapy and chemotherapy gives better results around the $50^{\text {th }}$ day than the use of surgery, radiotherapy and chemotherapy. This observation can be explained due to the fact that in our model, the use of surgery enhances the proliferation of the remaining tumour cell, because there are no more hypoxic tumour cells, and migrate further into the brain escaping the irradiated area. Yet you can observe that on the long run that there are less tumour cells in the 


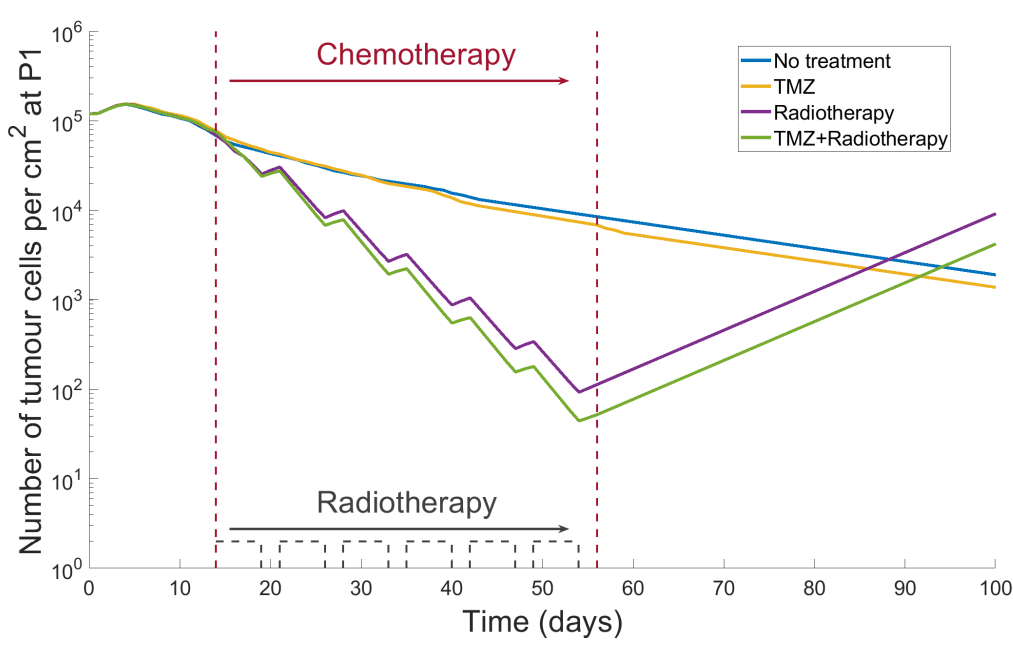

Fig. 7: Number of the tumour cells per $\mathrm{cm}^{2}$ at $P 1$ through time for different treatment schedules. The location of $P 1$ (the tumour core) is shown in the figure $3 \mathrm{~b}$. If performed, surgery is done on the first day, chemotherapy (TMZ) is administered from day 14 to day 56 and radiotherapy is administered 5 days out of 7 from day 14 to day 54 . Treatments using surgery are not displayed because no tumour cell remain after surgery at $P 1$.

brain when using surgery, TMZ and radiotherapy than TMZ and radiotherapy which implies a longer survival time when using all treatments.

\section{Discussion}

In all the simulations after the delivery of all the treatments, the tumour starts proliferating again until being slowed by the hypoxic tumour core. However the main criteria related to a patient death due to Glioblastoma is the total area occupied by tumour cells, so if tumour cells remain in the brain after the use of treatments then their proliferation induce the relapse of the patient and usually its death.

It must be remembered that all results presented in this work rely on the behaviour of our model, and so, cannot take every effects that would change impact the tumour growth. Indeed, in our model, if we wanted to enhance the death rate of tumour cells due to radiotherapy, we would increase the irradiation dose. However a higher dosage would impact the healthy cells in the brain that would not be able to repair their DNA-breaks as before. Also surgery in our model seems to amplify the spread of the remaining tumour cells. It would then be better to only use radiotherapy with chemotherapy as evidenced in the figure 6 but if surgery is not performed then there is a necrotic tumour core in the brain that could have negative impacts on the surrounding healthy tissues. For example the $\mathrm{O}_{2}$ delivery would decrease for the healthy tissues in favor of supplying the hypoxic tumour cells. Also we decided in this work to 


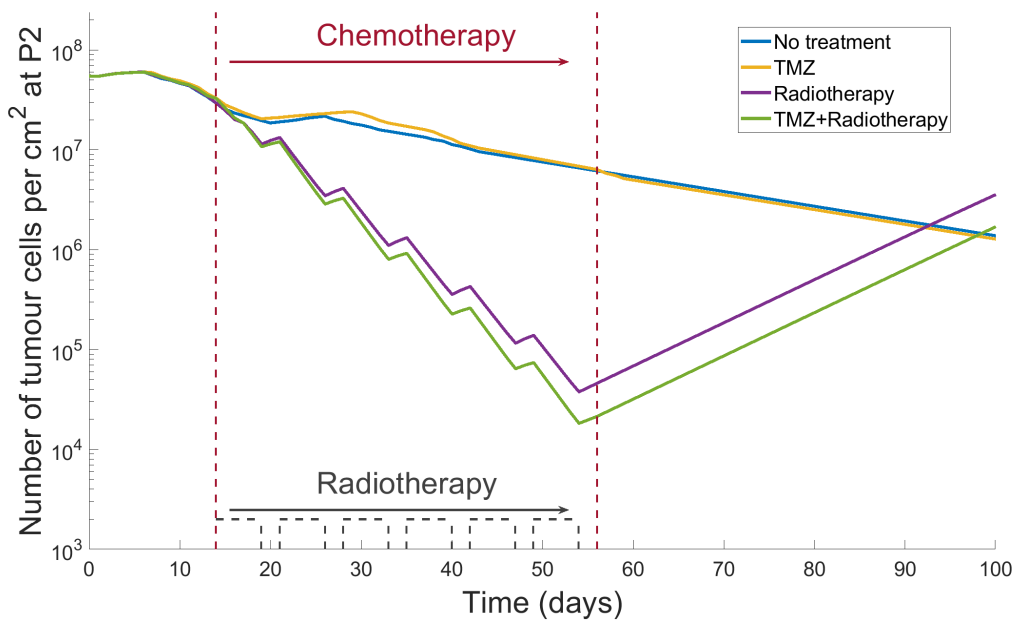

Fig. 8: Number of the tumour cells per $\mathrm{cm}^{2}$ at $P 2$ through time for different treatment schedules. The location of $P 2$ (the edema) is shown in the figure $3 \mathrm{~b}$. If performed, surgery is done on the first day, chemotherapy (TMZ) is administered from day 14 to day 56 and radiotherapy is administered 5 days out of 7 from day 14 to day 54 . Treatments using surgery are not displayed because no tumour cell remain after surgery at $P 2$.

consider only one population of tumour cells that react the same way to treatments. However some tumour cells can have random mutations that protect them against Temozolomide and radiotherapy. Those cells are not impacted by the treatments, except surgery, and are free to proliferate in the brain. It would then be necessary to consider two sub-populations of tumour cells according to the presence or not of the mutation that would not follow the exact same equation as (1a).

In this work we have shown that our model (1a)-(3) can perform simulations based on patient's MRIs and so, try to fit the growth of the Glioblastoma for that patient. Modifying the value of the different coefficients in (1a)-(1d) is the only way in our model to exhibit different growth behaviour like having a higher tumour proliferation, having a faster VEGF production or having a higher tumour diffusion rate. This approach works when the coefficients in (1a)-(1d) are known and well identified on a patient. Nowadays, a lot of information can be retrieved from the patient diagnosis: using immunohistochemistry-based algorithm (Orzan et al, 2020), analysing the extracellular vesicles situated in the glioblastoma micro-environment (Simon et al, 2020) or by determining the glioblastoma subtypes based on the OMS description (Louis et al, 2016). However those information are not linked explicitly to the different coefficients, and so, the coefficients have to be extrapolated from the information and then adapted to fit the growth of the patient tumour.

A solution to find the coefficients of a patient could be to set some coefficients as unknowns and write the system (1a)-(3) as an optimization problem based on the 


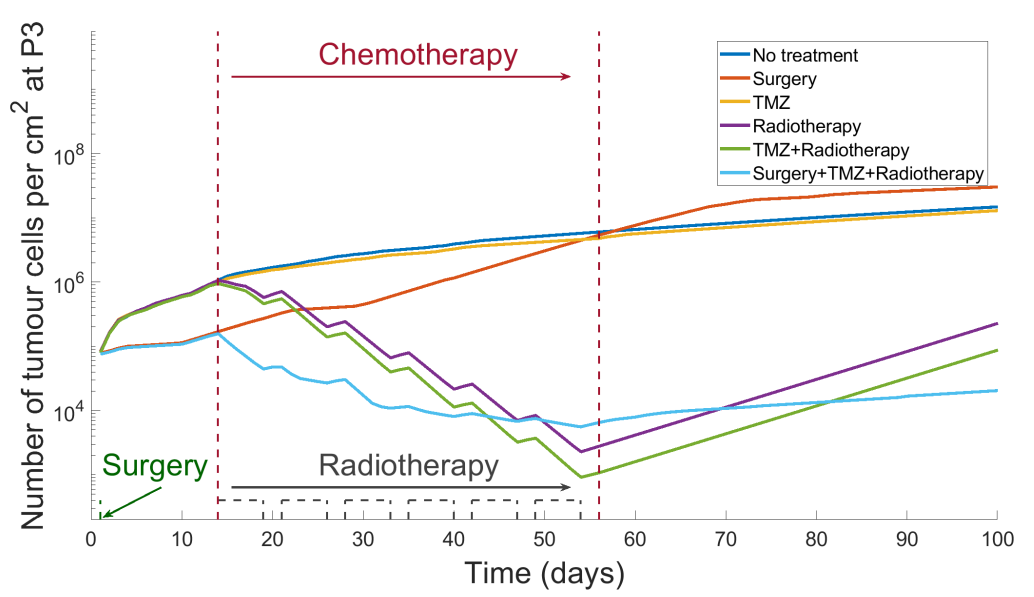

Fig. 9: Number of the tumour cells per $\mathrm{cm}^{2}$ at $P 3$ through time for different treatment schedules. The location of $P 3$ (the enhancing tumour body) is shown in the figure $3 \mathrm{~b}$. If performed, surgery is done on the first day, chemotherapy (TMZ) is administered from day 14 to day 56 and radiotherapy is administered 5 days out of 7 from day 14 to day 54 .

knowledge of the solution at different time steps. However this method shows weaknesses as there are more unknowns than equations which implies the need of more data that are not available from the diagnosis so we can not do simulations or predictions after the diagnosis. To solve this problem, we could not set the coefficients as unknowns but as temporal functions and use Kalman filter as in (Rochoux et al, 2018 ) to fit the model to the patient through time. This method allows to have a unique model that will adapt to each patient during the simulations.

\section{References}

Anderson ARA (2005) A hybrid mathematical model of solid tumour invasion: the importance of cell adhesion. Mathematical Medicine and Biology: A Journal of the IMA 22:163-186, DOI https://doi.org/10.1093/imammb/dqi005

ANOCEF (2018) Référentiel glioblastome (grade iv oms). URL https: //www. onco-grandest.fr/wp-content/uploads/2020/07/anocef_ referentiel_glioblastome2018.pdf

Bakas S, Akbari H, Sotiras A, Bilello M, Rozycki M, Kirby JS, Freymann JB, Farahani K, Davatzikos C (2017) Advancing the cancer genome atlas glioma mri collections with expert segmentation labels and radiomic features. Sci Data 4(170117), DOI https://doi.org/10.1038/sdata.2017.117

Banerjee S, Khajanchi S, Chaudhuri S (2015) A mathematical model to elucidate brain tumor abrogation by immunotherapy with $\mathrm{t} 11$ target structure. PLoS ONE 10(5), DOI https://doi.org/10.1371/journal.pone.0123611 
Basanta D, Scott JG, Rockne R, Swanson KR, Anderson ARA (2011) The role of idh1 mutated tumour cells in secondary glioblastomas: an evolutionary game theoretical view. Phys Biol 8(1), DOI https://doi.org/10.1088/1478-3975/8/1/015016

Cancès C, Guichard C (2016) Convergence of a nonlinear entropy diminishing control volume finite element scheme for solving anisotropic degenerate parabolic equations. Math Comp 85:549-580, DOI https://doi.org/10.1090/mcom/2997

Cancès C, Ibrahim M, Saad M (2017) Positive nonlinear cvfe scheme for degenerate anisotropic keller-segel system. The SMAI journal of computational mathematics 3:1-28, DOI https://doi.org/10.5802/smai-jcm.18

Curtin L, Hawkins-Daarud A, van der Zee KG, Swanson KR, Owen MR (2020) Speed switch in glioblastoma growth rate due to enhanced hypoxia-induced migration. Bull Math Biol 82(43), DOI https://doi.org/10.1007/s11538-020-00718-x

Enderling H, Chaplain MAJ, Hahnfeldt P (2010) Quantitative modeling of tumor dynamics and radiotherapy. Acta Biotheor 58:341-353, DOI https://doi.org/10.1007/ s10441-010-9111-z

Foucher F, Ibrahim M, Saad M (2018) Convergence of a positive nonlinear control volume finite element scheme for solving an anisotropic degenerate breast cancer development model. Computers \& Mathematics with Applications 76(3):551-578, DOI https://doi.org/10.1016/j.camwa.2018.04.037

Hinow P, Gerlee P, McCawley LJ, Quaranta V, Ciobanu M, Wang S, Graham JM, Ayati BP, Claridge J, Swanson KR, Loveless M, Anderson ARA (2009) A spatial model of tumor-host interaction: Application of chemotherapy. Mathematical Biosciences \& Engineering 6(3):521-546, DOI 10.3934/mbe.2009.6.521

Jbabdi S, Mandonnet E, Duffau H, Capelle L, Swanson KR, Pélégrini-Issac M, Guillevin R, Benali H (2005) Simulation of anisotropic growth of low-grade gliomas using diffusion tensor imaging. Magnetic Resonance in Medecine 53:616 624, DOI https://doi.org/10.1002/mrm.20625

JR S (1996) Triangle: Engineering a 2d quality mesh generator and delaunay triangulator. Lecture Notes in Computer Science 1148, DOI https://doi.org/10.1007/ $\mathrm{BFb} 0014497$

Kamran N, Alghamri MS, Nunez FJ, Shah D, Asad AS, Candolfi M, Altshuler D, Lowenstein PR, Castro MG (2018) Current state and future prospects of immunotherapy for glioma. Immunotherapy 10(4), DOI https://doi.org/10.2217/ imt-2017-0122

Kim WY, Lee HY (2009) Brain angiogenesis in developmental and pathological processes: mechanism and therapeutic intervention in brain tumors. FEBS journal 276:4653-4664, DOI 10.1111/j.1742-4658.2009.07177.x

Kim Y, Lawler S, ONowicki M, Chiocca EA, Friedman A (2009) A mathematical model for pattern formation of glioma cells outside the tumor spheroid core. Journal of Theoretical Biology 260(3):359-371, DOI https://doi.org/10.1016/j.jtbi. 2009.06.025

KJPaintera, THillen (2013) Mathematical modelling of glioma growth: The use of diffusion tensor imaging (dti) data to predict the anisotropic pathways of cancer invasion. Journal of Theoretical Biology 323:25-39, DOI https://doi.org/10.1016/ j.jtbi.2013.01.014 
Lai X, Friedman A (2020) Mathematical modeling of cancer treatment with radiation and pd-11 inhibitor. Sci China Math 63:465-484, DOI https://doi.org/10.1007/ s11425-019-1648-6

Lim M, Xia Y, Bettegowda C, Weller M (2018) Current state of immunotherapy for glioblastoma. Nat Rev Clin Oncol 15:422-442, DOI https://doi.org/10.1038/ s41571-018-0003-5

Louis DN, Perry A, Reifenberger G, von Deimling A, Figarella-Branger D, Cavenee WK, Ohgaki H, Wiestler OD, Kleihues P, Ellison DW (2016) The 2016 world health organization classification of tumors of the central nervous system: a summary. Acta Neuropathol 131:803-820, DOI https://doi.org/10.1007/ s00401-016-1545-1

Lundervold AS, Lundervold A (2019) An overview of deep learning in medical imaging focusing on mri. Zeitschrift für Medizinische Physik 29(2):102-127, DOI https://doi.org/10.1016/j.zemedi.2018.11.002

Mantzaris NV, Webb S, Othmer HG (2004) Mathematical modeling of tumorinduced angiogenesis. J Math Biol 49:11-187, DOI https://doi.org/10.1007/ s00285-003-0262-2

Nilsson P, Thames HD, Joiner MC (1990) A generalized formulation of the 'incomplete-repair' model for cell survival and tissue response to fractionated low dose-rate irradiation. Int J Radiat Biol 57:127-142, DOI 10.1080/ 09553009014550401

Orzan F, Pagani F, Cominelli M, Triggiani L, Calza S, Bacco FD, Medicina D, Balzarini P, Panciani PP, Liserre R, Buglione M, Fontanella MM, Medico E, Galli R, Isella C, Boccaccio C, Poliani PL (2020) A simplified integrated molecular and immunohistochemistry-based algorithm allows high accuracy prediction of glioblastoma transcriptional subtypes. Lab Invest 100:1330-1344, DOI https://doi.org/10.1038/s41374-020-0437-0

Papadogiorgaki M, Koliou P, Kotsiakis X, Zervakis ME (2013) Mathematical modelling of spatio-temporal glioma evolution. Theor Biol Med Model 10(47), DOI https://doi.org/10.1186/1742-4682-10-47

Pati S, Singh A, Rathore S, Gastounioti A, Bergman M, Ngo P, Ha SM, Bounias D, Minock J, Murphy G, Li H, Bhattarai A, Wolf A, Sridaran P, Kalarot R, Akbari H, Sotiras A, Thakur SP, Verma R, Shinohara RT, Yushkevich P, Kontos YFD, Davatzikos C, Bakas S (2020) The cancer imaging phenomics toolkit (captk): Technical overview. Lecture Notes in Computer Science 11993, DOI https://doi.org/10.1007/978-3-030-46643-5_38

Powathil G, Kohandel M, Sivaloganathan S, Oza A, Milosevic M (2007) Mathematical modeling of brain tumors: effects of radiotherapy and chemotherapy. Phys Med Biol 52(11):3291-3306, DOI 10.1088/0031-9155/52/11/023

Rochoux M, Collin A, Zhang C, Trouvé A, Lucor D, Moireau P (2018) Front shape similarity measure for shape-oriented sensitivity analysis and data assimilation for eikonal equation. ESAIM: ProcS 63:258-279, DOI https://doi.org/10.1051/proc/ 201863258

Schugart RC, Friedman A, Zhao R, Sen CK (2008) Wound angiogenesis as a function of tissue oxygen tension: A mathematical model. PNAS 105:2628-2633, DOI https://doi.org/10.1073/pnas.0711642105 
Shewchuk JR (2002) Delaunay refinement algorithms for triangular mesh generation. Computational Geometry 22:21-74, DOI https://doi.org/10.1016/S0925-7721(01) 00047-5

Simon T, Jackson E, Giamas G (2020) Breaking through the glioblastoma microenvironment via extracellular vesicles. Oncogene 39:4477-4490, DOI https://doi. org/10.1038/s41388-020-1308-2

Spill F, Guerrero P, Alarcon T, Maini PK, Byrne HM (2015) Mesoscopic and continuum modelling of angiogenesis. J Math Biol 70:485-532, DOI https://doi.org/10. 1007/s00285-014-0771-1

Stein AM, Demuth T, Mobley D, Berens M, Sander LM (2007) A mathematical model of glioblastoma tumor spheroid invasion in a three-dimensional in vitro experiment. Biophysical journal 92(1):356-365, DOI https://doi.org/10.1529/ biophysj.106.093468

Stupp R, Mason WP, van den Bent MJ, Weller M, Fisher B, Taphoorn MJ, Belanger K, Brandes AA, Marosi C, Bogdahn U, Curschmann J, Janzer RC, Ludwin SK, Gorlia T, Allgeier A, Lacombe D, Cairncross JG, Eisenhauer E, Mirimanoff RO (2005) Radiotherapy plus concomitant and adjuvant temozolomide for glioblastoma. N Engl J Med 352:987-996, DOI 10.1056/NEJMoa043330

Subramanian S, Gholami A, Biros G (2019) Simulation of glioblastoma growth using a 3d multispecies tumor model with mass effect. J Math Biol 79:941-967, DOI https://doi.org/10.1007/s00285-019-01383-y

TCIA (2018) Radiology data from the clinical proteomic tumor analysis consortium glioblastoma multiforme [cptac-gbm] collection [data set]. DOI https://doi.org/ 10.7937/k9/tcia.2018.3rje41q1, URL https : //wiki . cancerimagingarchive . net/display/Public/CPTAC-GBM

Tracqui P, Cruywagen GC, Woodward DE, Bartoo GT, Murray JD, Jr ECA (1995) A mathematical model of glioma growth: the effect of chemotherapy on spatiotemporal growth. Cell Proliferation in basic and clinical sciences 28(1):17-31, DOI https://doi.org/10.1111/j.1365-2184.1995.tb00036.x

Travasso RDM, Poiré EC, Castro M, Rodrguez-Manzaneque JC, HernándezMachado A (2011) Tumor angiogenesis and vascular patterning: A mathematical model. PLoS ONE 6(5), DOI https://doi.org/10.1371/journal.pone.0019989

Vilanova G, Colominas I, Gomez H (2017) A mathematical model of tumour angiogenesis: growth, regression and regrowth. J R Soc 14(126), DOI https://doi.org/10. 1098/rsif.2016.0918

Villanueva-Meyer JE, Mabray MC, Cha S (2017) Current clinical brain tumor imaging. Neurosurgery 81(3):397-415, DOI https://doi.org/10.1093/neuros/nyx103

Woodward DE, Cook J, Tracqui P, Cruywagen GC, Murray JD, Jr ECA (1996) A mathematical model of glioma growth: the effect of extent of surgical resection. Cell Proliferation in basic and clinical sciences 29(6):269-288, DOI https://doi. org/10.1111/j.1365-2184.1996.tb01580.x

\section{A Supplementary Information}


Table 5: Description of all variables used in this paper

\begin{tabular}{|c|c|}
\hline variables & descriptions \\
\hline$u$ & $\begin{array}{l}\text { concentration in tumour cells divided by the maximum cell concentration allowed in } \\
\text { tissues }\end{array}$ \\
\hline$c$ & concentration in $O_{2}$ in $\mu \mathrm{mol} \cdot \mathrm{cm}^{-2}$ \\
\hline$u_{e}$ & $\begin{array}{l}\text { concentration in endothelial cells divided by the maximum cell concentration allowed } \\
\text { in tissues }\end{array}$ \\
\hline$v$ & concentration in Vascular Endothelial Growth Factor in $\mu \mathrm{mol} \cdot \mathrm{cm}^{-2}$ \\
\hline$u_{T}$ & $\begin{array}{l}\text { total concentration of cell populations divided by the maximum cell concentration } \\
\text { allowed in tissues, it is the sum of } u \text { and } u_{e}\end{array}$ \\
\hline$u_{K}^{n}$ & numerical approximation of $u$ at the vertex $x_{K}$ and the time $t_{n}$ \\
\hline$c_{K}^{n}$ & numerical approximation of $c$ at the vertex $x_{K}$ and the time $t_{n}$ \\
\hline$u_{e, K}^{n}$ & numerical approximation of $u_{e}$ at the vertex $x_{K}$ and the time $t_{n}$ \\
\hline$v_{K}^{n}$ & numerical approximation of $v$ at the vertex $x_{K}$ and the time $t_{n}$ \\
\hline$u_{T, K}^{n}$ & numerical approximation of $u_{T}$ at the vertex $x_{K}$ and the time $t_{n}$ \\
\hline$\Lambda_{K L}^{(1)}$ & numerical approximation of the diffusion flux $\Lambda_{1}(x) \vec{n}$ on the edge $\sigma_{K L}$ \\
\hline$\Lambda_{K L}^{(3)}$ & numerical approximation of the diffusion flux $\Lambda_{3}(x) \vec{n}$ on the edge $\sigma_{K L}$ \\
\hline$D_{K L}^{(2)}$ & numerical approximation of the diffusion flux $D_{2} \vec{n}$ on the edge $\sigma_{K L}$ \\
\hline$D_{K L}^{(4)}$ & numerical approximation of the diffusion flux $D_{4} \vec{n}$ on the edge $\sigma_{K L}$ \\
\hline$a_{K L}^{n}$ & numerical approximation of the cell-dependant diffusion $a(u)$ on the edge $\sigma_{K L}$ \\
\hline$\mu_{K L}^{n}$ & numerical approximation of the cell-dependant chemotaxis $\mu_{1}(u)$ on the edge $\sigma_{K L}$ \\
\hline$\eta_{K L}^{n}$ & numerical approximation of $\eta(c)$ on the edge $\sigma_{K L}$ \\
\hline$\tilde{a}_{K L}^{n}$ & numerical approximation of the cell-dependant diffusion $a\left(u_{e}\right)$ on the edge $\sigma_{K L}$ \\
\hline$\tilde{\mu}_{K L}^{n}$ & numerical approximation of the cell-dependant chemotaxis $\mu_{3}\left(u_{e}\right)$ on the edge $\sigma_{K L}$ \\
\hline $\bar{\eta}_{K L}^{n}$ & numerical approximation of $\eta(v)$ on the edge $\sigma_{K L}$ \\
\hline
\end{tabular}


Table 6: Description of all coefficients used in this paper

\begin{tabular}{|c|c|c|}
\hline Coefficients & Descriptions & Units \\
\hline$T_{f}$ & final time used in simulations & day \\
\hline$\rho_{1}$ & growth rate of tumour cells & day $^{-1}$ \\
\hline$\beta_{1}$ & apoptosis rate of tumour cells & day $^{-1}$ \\
\hline$\alpha_{2}$ & production rate of $O_{2}$ by endothelial cells & $\mu \mathrm{mol} \cdot$ day $^{-1}$ \\
\hline$\beta_{2}$ & degradation rate of $\mathrm{O}_{2}$ & day $^{-1}$ \\
\hline$\gamma_{2}$ & consumption rate of $\mathrm{O}_{2}$ by tumour cells & day $^{-1}$ \\
\hline$\rho_{3}$ & growth rate of endothelial cells & day $^{-1}$ \\
\hline$\beta_{3}$ & apoptosis rate of endothelial cells & day $^{-1}$ \\
\hline$\alpha_{4}$ & production rate of VEGF by endothelial cells & $\mu \mathrm{mol} \cdot$ day $^{-1}$ \\
\hline$\beta_{4}$ & degradation rate of VEGF & day $^{-1}$ \\
\hline$\gamma_{4}$ & consumption rate of VEGF by endothelial cells & day $^{-1}$ \\
\hline$D_{2}$ & isotropic diffusion matrix of $O_{2}$ in the brain & $\left(\mathrm{cm}^{2} \cdot \text { day }^{-1}\right)^{2 \times 2}$ \\
\hline$D_{4}$ & isotropic diffusion matrix of VEGF in the brain & $\left(\mathrm{cm}^{2} \cdot \text { day }^{-1}\right)^{2 \times 2}$ \\
\hline$c_{\text {hypo }}$ & threshold under which cells are hypoxic & $\mu \mathrm{mol} \cdot \mathrm{cm}^{-2}$ \\
\hline$c_{\text {necro }}$ & threshold under which cells necrose & $\mu \mathrm{mol} \cdot \mathrm{cm}^{-2}$ \\
\hline$u_{\max }$ & maximum tissue capacity & cells $\cdot \mathrm{cm}^{-2}$ \\
\hline$h_{\mathscr{T}}$ & size of the triangulation $\mathscr{T}$ & $\mathrm{cm}^{2}$ \\
\hline$\theta_{\mathscr{T}}$ & regularity of the triangulation $\mathscr{T}$ & \\
\hline$\delta t$ & time step used in simulations & day \\
\hline$t_{n}$ & nth discrete time step value & day \\
\hline$D_{\text {che }}$ & death rate of tumour cells induced by chemotherapy & day $^{-1}$ \\
\hline$R_{\text {eff }}$ & death rate of tumour cells induced by radiotherapy & day $^{-1}$ \\
\hline$N_{\text {frac }}$ & number of radiotherapy fractions administered in a day & \\
\hline$D_{\text {rad }}$ & dosage per fraction & $G_{\gamma}$ \\
\hline$\tau$ & irradiation time for a fraction & $\min$ \\
\hline$\Delta \tau$ & time between consecutive irradiations & $\min$ \\
\hline$\mu$ & DNA damaged rate & $\min ^{-1}$ \\
\hline$\alpha$ & sensitivity parameter & day $^{-1} \cdot G_{\gamma}^{-1}$ \\
\hline$\beta$ & sensitivity parameter & $\mathrm{day}^{-1} \cdot G_{\gamma}^{-2}$ \\
\hline \multicolumn{3}{|l|}{$\varphi$} \\
\hline$d_{w m}$ & diffusion rate of cells in white matter & $\mathrm{cm}^{2} \cdot \mathrm{day}^{-1}$ \\
\hline$d_{g m}$ & diffusion rate of cells in grey matter & $\mathrm{cm}^{2} \cdot$ day $^{-1}$ \\
\hline$d_{p s}$ & diffusion rate of cells in the post surgical area & $\mathrm{cm}^{2} \cdot \mathrm{day}^{-1}$ \\
\hline$d_{p}$ & diffusion rate of $O_{2}$ and VEGF in the brain & $\mathrm{cm}^{2} \cdot$ day $^{-1}$ \\
\hline tolImplicit & threshold to stop the computation using $\tau^{(n)}(\cdot)$ & \\
\hline tolNewton & threshold to stop the Newton algorithm & \\
\hline tolGradient & threshold to stop the conjugate gradient algorithm & \\
\hline r & $\begin{array}{l}\text { efficiency rate between radiotherapy only and radiotherapy with } \\
\text { chemotherapy }\end{array}$ & \\
\hline$\lambda_{1}$ & chemotaxis coefficients of tumour cells & $\mathrm{cm}^{2} \cdot \mu \mathrm{mol}^{-1} \cdot \mathrm{day}^{-1}$ \\
\hline$\lambda_{3}$ & chemotaxis coefficients of endothelial cells & $\mathrm{cm}^{2} \cdot \mu \mathrm{mol}^{-1} \cdot$ day $^{-1}$ \\
\hline
\end{tabular}


Table 7: Description of all functions used in this paper

\begin{tabular}{ll}
\hline functions & descriptions \\
\hline$\Lambda_{1}(\cdot)$ & medium-dependent diffusion matrix of tumour cells \\
$\Lambda_{3}(\cdot)$ & medium-dependent diffusion matrix of endothelial cells \\
$a(\cdot)$ & cell-dependant diffusion function of cells \\
$\chi_{1}(\cdot)$ & cell-dependent chemotaxis function of tumour cells \\
$\chi_{3}(\cdot)$ & cell-dependent chemotaxis function of endothelial cells \\
$\mu_{1}(\cdot)$ & ratio function between $\chi_{1}(\cdot)$ and $a(\cdot)$ \\
$\mu_{3}(\cdot)$ & ratio function between $\chi_{3}(\cdot)$ and $a(\cdot)$ \\
$f(\cdot)$ & cells-dependent reproduction functions of tumour and endothelial cells \\
$T_{\text {treat }}(\cdot, \cdot)$ & time and cell-dependent treatment map \\
$T_{\text {chemo }}(\cdot, \cdot)$ & time and cell-dependent treatment map modeling chemotherapy \\
$T_{\text {radio }}(\cdot, \cdot)$ & time and cell-dependent treatment map modeling radiotherapy \\
$g(\cdot)$ & $O_{2}$-dependent function used for VEGF production under hypoxia \\
$h(\cdot)$ & $O_{2}$-dependent function used for tumour cells mitosis \\
$\vec{n}$ & unit normal vector on a boundary \\
$u_{0}$ & initial tumour cells concentration in the brain \\
$c_{0}$ & initial $O_{2}$ concentration in the brain \\
$u_{e_{0}}$ & initial endothelial cells concentration in the brain \\
$v_{0}$ & initial VEGF concentration in the brain \\
$\eta(\cdot)$ & function used to ensure positivity of $O_{2}$ and VEGF concentration \\
$p(\cdot)$ & function used to ensure positivity of $O_{2}$ and VEGF concentration \\
$\mathscr{A}(\cdot)$ & primitive function of $a(\cdot)$ \\
$\xi(\cdot)$ & primitive function of $\sqrt{a(\cdot)}$ \\
$(\Phi)_{I}$ & canonical basis of $\mathscr{H}_{\mathscr{T}}$ \\
$\mu_{\uparrow}$ & primitive function of $\left(\mu^{\prime}(\cdot)\right)^{+}$ \\
$\mu_{\downarrow}$ & primitive function of $-\left(\mu^{\prime}(\cdot)\right)^{-}$ \\
$g_{\text {rad }}(\cdot)$ & functional where the kernel gives the solution of the main system \\
$h_{n}(\cdot)$ & function used for modeling radiotherapy \\
$\tau^{(n)}(\cdot)$ & sequence of functions used to find a solution of an implicit scheme from a semi- \\
$k_{c}(\cdot)$ & implicit scheme \\
$k_{r}(\cdot)$ & time-dependent function equal to 1 when chemotherapy is administered \\
$k_{\text {chemo }}(\cdot)$ & time-dependent function equal to 1 when radiotherapy is performed \\
& used \\
&
\end{tabular}


Table 8: Description of all spaces used in this paper

\begin{tabular}{ll}
\hline Spaces & descriptions \\
\hline$\Omega$ & working space based on a 2D-slice of brain delimited by the skull \\
$\partial \Omega$ & border of $\Omega$ \\
$\mathscr{T}$ & conforming triangulation on $\Omega$ \\
$\mathscr{E}$ & set of edges from $\mathscr{T}$ \\
$v$ & set of vertices from $\mathscr{T}$ \\
$\mathscr{E}_{K}$ & subset of $\mathscr{E}$ of $K$ as a vertex \\
$T_{K}$ & set of triangles having $K$ as a vertex \\
$\mathscr{M}$ & dual mesh constructed from $\mathscr{T}$ \\
$\mathscr{H}_{\mathscr{T}}$ & the $\mathbb{P}_{1}(\mathbb{R})$ finite element space \\
$\chi_{\mathscr{K}}$ & the discrete control volumes space \\
$\mathscr{T}_{K L}^{n+1}$ & interval of values between $u_{K}^{n+1}$ and $u_{L}^{n+1}$ \\
$\mathbb{Q}_{K L}^{n+1}$ & interval of values between $c_{K}^{n+1}$ and $c_{L}^{n+1}$ \\
$\mathscr{J}_{K L}^{n+1}$ & interval of values between $u_{e, K}^{n+1}$ and $u_{e, L}^{n+1}$ \\
$J_{K L}^{n+1}$ & interval of values between $v_{K}^{n+1}$ and $v_{L}^{n+1}$ \\
$E$ & set of vectors used for proof \\
\hline
\end{tabular}

Table 9: Description of all mesh components used in this paper

\begin{tabular}{ll}
\hline Mesh components & descriptions \\
\hline$h_{t}$ & the diameter of the triangle $t$ \\
$\rho_{t}$ & the diameter of the incircle of the triangle $t$ \\
$x_{K}$ & the coordinates of the vertex $K$ \\
$\sigma_{K L}$ & the edge joining the vertex $K$ and $L$ \\
$\omega_{K}$ & the dual element constructed around the vertex $K$ \\
$m_{K}$ & the 2-dimensional Lebesgue measure of $\omega_{K}$ \\
\hline
\end{tabular}

